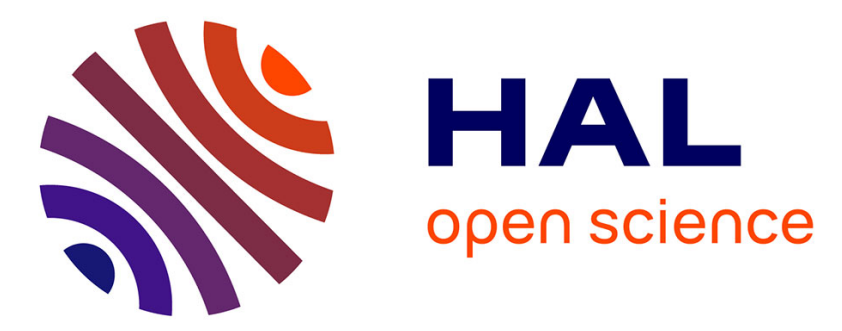

\title{
pH-Driven Self-Assembly of Acidic Microbial Glycolipids
}

Niki Baccile, Mohamed Selmane, Patrick Le Griel, Sylvain Prévost, Javier

Perez, Christian V. Stevens, Elisabeth Delbeke, Susanne Zibek, Michael Guenther, Wim Soetaert, et al.

\section{- To cite this version:}

Niki Baccile, Mohamed Selmane, Patrick Le Griel, Sylvain Prévost, Javier Perez, et al.. pHDriven Self-Assembly of Acidic Microbial Glycolipids. Langmuir, 2016, 32 (25), pp.6343-6359. 10.1021/acs.langmuir.6b00488 . hal-01359051

\section{HAL Id: hal-01359051 https: / hal.sorbonne-universite.fr/hal-01359051}

Submitted on 3 Feb 2017

HAL is a multi-disciplinary open access archive for the deposit and dissemination of scientific research documents, whether they are published or not. The documents may come from teaching and research institutions in France or abroad, or from public or private research centers.
L'archive ouverte pluridisciplinaire HAL, est destinée au dépôt et à la diffusion de documents scientifiques de niveau recherche, publiés ou non, émanant des établissements d'enseignement et de recherche français ou étrangers, des laboratoires publics ou privés. 
IMPORTANT NOTE : Please be aware that slight modifications occurring after Proof correction may occur between this version of the manuscript and the version on the Publisher's website-

\title{
pH-driven self-assembly of acidic microbial glycolipids
}

Niki Baccile, ${ }^{1 *}$ Mohamed Selmane, ${ }^{1}$ Patrick Le Griel, ${ }^{1}$ Sylvain Prévost, ${ }^{2}$ Javier Perez, ${ }^{3}$ Christian V. Stevens, ${ }^{4}$ Elisabeth Delbeke, ${ }^{4}$ Susanne Zibek, ${ }^{5}$ Michael Guenther, ${ }^{5}$ Wim Soetaert, ${ }^{6}$ Inge N. A. Van Bogaert, ${ }^{6}$ Sophie Roelants ${ }^{6,7}$

1 Sorbonne Universités, UPMC Univ Paris 06, CNRS, Collège de France UMR 7574, Chimie de la Matière Condensée de Paris, UMR 7574, F-75005 Paris, France. Corresponding author : niki.baccile@upmc.fr

2 ESRF - The European Synchrotron, High Brilliance Beamline ID02, 38043 Grenoble, France

3 SWING, Synchrotron Soleil, BP 48, 91192 Gif-sur-Yvette, France

4 SynBioC, Department of Sustainable Organic Chemistry and Technology, Ghent University, Ghent, Belgium

5 Fraunhofer Institute for Interfacial Engineering and Biotechnology IGB, 70569 Stuttgart, Germany

6 InBio, Department of Biochemical and Microbial Technology, Faculty of Bioscience Engineering, Ghent University, Coupure Links 653, 9000, Ghent, Belgium

7 Bio Base Europe Pilot Plant, Rodenhuizekaai 1, 9042 Gent, Belgium

\begin{abstract}
Microbial glycolipids are a class of well-known compounds, but their self-assembly behaviour is still not well understood. While the free carboxylic acid end-group makes some of them interesting stimuli-responsive compounds, the sugar hydrophilic group and the nature of the fatty acid chain make the understanding of their self-assembly behaviour in water not easy and highly unpredictable. Using cryo-Transmission Electron Microscopy (TEM) and both $\mathrm{pH}$-dependent in-situ and ex-situ Small Angle X-ray Scattering (SAXS), we demonstrate that the aqueous self-assembly at room temperature (RT) of a family of $\beta$-D-glucose microbial glycolipids bearing a saturated and monounsaturated C18 fatty acid chain cannot be explained on the simple basis of the well-known packing parameter. Using the "pH-jump" process we find that the molecules bearing a monosaturated fatty acid forms vesicles below
\end{abstract}


pH 6.2, as expected, but the derivative with a saturated fatty acid form infinite bilayer sheets below $\mathrm{pH}$ 7.8, instead of vesicles. We show that this behaviour can be explained on the different bilayer membrane elasticity as a function of temperature. Membranes are either flexible or stiff for experiments performed at a temperature respectively above or below the typical melting point, $\mathrm{T}_{\mathrm{M}}$, of the lipidic part of each compound. Finally, we also show that the disaccharide-containing acidic cellobioselipid forms a majority of chiral fibers, instead of the expected micelles.

\section{Introduction}

Glycolipids are a general class of compounds composed of a sugar and lipid moieties covalently bonded together. Sugar-based amphiphiles are a class of molecules which attract much attention amongst others due to their astonishing self-assembly properties in water. ${ }^{1,2,3,4,5,6}$ Their biobased and renewable origin, good biodegradability and the variety (micelles, vesicles, fibers, bilayers, chiral fibers) of supramolecular structures they can form, make them valuable substitutes for more classical/synthetic types of surfactants, like alkylammonium, alkylsulfates and block copolymers. The importance of carbohydrates in glycobiology and medicine, ${ }^{7}$ their complex physico-chemical behaviour occurring via oriented hydrogen bonding ${ }^{8,9}$ and the richness of carbohydrate chemistry are some of the most interesting features that these compounds can potentially express. Furthermore, most glycolipids have additional chemical functions like acetylenic, carboxylic, ${ }^{4,5}$ hydroxy ${ }^{4}$ in their aliphatic moiety, thus increasing the level of complexity and functionality of these compounds and making them promising precursors for application in advanced material science. ${ }^{10,11}$ However, even their simplest structural form hides an underlying complexity in terms of collective supramolecular behaviour.

Simple forms of glycolipids bearing a mono-, di- or tri-saccharide connected to the aliphatic chain via an acetal can predictably form micellar aggregates. ${ }^{12,13}$ However, introduction of amide or phenyl groups as linkers between the carbohydrate and the lipid drives the systems towards the formation of more complex structures like nanotubes ${ }^{14}$ or coiled fibers. ${ }^{2}$ On the contrary, complex compounds like glycosphingolipids, glycosylated derivatives of aliphatic amino alcohols, can aggregate into micelles or, most importantly, contribute to the stability and functionality of biological plasma membranes. ${ }^{15}$ Even if the amount of examples in which the self-assembly behaviour of glycolipids is reported in the literature is very large, prediction of the type of aggregate is still difficult, because of the large 
number of factors influencing the self-assembly process. These can be divided into two categories, physico-chemical and structural. The former refers to experimental conditions, typically temperature, $\mathrm{pH}$, ionic force but also process, like rate of heating/cooling or $\mathrm{pH}$ change, order of mixing, etc. These factors are known to have an important influence on the self-assembly of most amphiphiles, including glycolipids. It was shown, for instance, that the rate of $\mathrm{pH}^{16}$ or temperature ${ }^{17}$ jump in vesicle-forming ethylene glycol-based amphiphiles, or in phospholipids, influences the size and type of aggregation: slow variation in temperature or in $\mathrm{pH}$ induces the formation of larger, or more aggregated, vesicles. In general, it was shown that, even if self-assembly properties of amphiphiles (aggregation number, critical micellar concentration, size and shape of micelles) can be predicted at equilibrium using thermodynamic arguments, ${ }^{18}$ kinetics of aggregation and shape evolution should not be disregarded, because of the non-equilibrium phenomena. Therefore, in some cases it can be necessary to explain unpredictable facts, as it was demonstrated for instance in micelle-tovesicle transition mechanisms in lecithin/bile salts systems. ${ }^{19}$ The second category refers to the actual chemical structure of the glycolipids, e.g. the number and type of sugar units, the type of glycosidic bond ( $\alpha-$, $\beta$-, position), the chemical nature of the sugar-lipid linker, the type of lipid including size, the presence of branching, the number and position of unsaturation, the presence of additional chemical groups, etc. The quantitative ${ }^{18}$ and qualitative (e.g., the notion of packing parameter ${ }^{20}$ thermodynamic treatment of these systems could certainly be of help to predict the self-assembly behaviour of such compounds, as this was among others demonstrated in carbohydrate-based gemini amphiphiles. ${ }^{21}$ However, a simple geometrical packing model cannot explain many experimental facts, as also shown on phospholipid and/or ethylene glycol-based systems. The notion of packing parameter introduced by Israelachvili, Mitchell and Ninham ${ }^{20}$ comes from a simple geometric static observation, while a more precise description of actual aggregation processes can involve dynamics. In this case, one can explain the self-assembly process by associating the amphiphile aggregate to a membrane described by two principal radii of curvature, which define the mean and gaussian curvatures of the membrane itself. Predictions can then be made based on the evolution of the membrane curvature $22,17,23$ in relation to both the amphiphile geometry and the surrounding physico-chemical conditions. ${ }^{16,19}$ For instance, it was shown that membrane fluidity in the preparation of nanoliposomes from phospholipids depends both the nature of the lipid moiety (saturation vs. monounsaturation), headgroup size and charge (neutral vs. charged), but also on the temperature at which the experiments are carried out. ${ }^{16}$ The self-assembly of saturated acidic sophorolipids, a family of microbial glycolipids, can be 
strongly influenced by its physico-chemical history and should be treated as a system out of equilibrium: if the compound is directly dissolved in water at neutral $\mathrm{pH},{ }^{24}$ flat sheets are formed; however, if the compound is initially treated at basic $\mathrm{pH}$ to increase its water solubility before being stabilized at neutral $\mathrm{pH}$, stable twisted ribbons are formed. ${ }^{25}$

In a series of recent works, we have shown how two $\mathrm{pH}$-responsive structurallysimilar sophorolipids, ${ }^{26}$ only differing in the presence or absence of a double bond, show a drastically different behaviour in water at $\mathrm{pH}$ values below 7: the monounsaturated acidic sophorolipid forms micelles ${ }^{27,28}$ while the saturated congener forms twisted ribbons. ${ }^{25}$ Interestingly, under basic $\mathrm{pH}$ conditions these two compounds show exactly the same behaviour, forming a mixture of micelles and aggregated nanoscale platelets. ${ }^{29}$ These observations are both hard to predict and to explain simply based on the difference between the lipid moiety (monounsaturated vs. saturated): both compounds are expected to have a very similar packing parameter, which is calculated and detailed further in the discussion section of this work and expected to be in the vicinity of 0.3. Under these conditions, sophorolipids are expected to form stable micelles, but not flat structures like ribbons, of which the packing parameter is unitary. These observations make it necessary to shed more light on this topic.

To do so, in this paper we study three microbial glycolipid compounds. The first two are two glucolipids congeners, the structure of which is exactly the same as sophorolipids, except for the number of sugars in the hydrophilic headgroup: sophorolipids are composed of sophorose, a $\beta$-D-glucose $\beta(1,2)$ disaccharide, while the glucolipids are composed of one single $\beta$-D-glucose unit. The self-assembly properties of these glucolipids have never been studied before, although their structure is comparable (except for the type of sugar-lipid linker) to similar nanotube-forming glycolipids obtained through synthetic chemistry. ${ }^{30}$ The comparison between their self-assembly behaviour to that of sophorolipids will help to better understand the properties of this class of compounds and in particular the role of both the sugar headgroup and fatty acid chain in the assembly behaviour. The third type of glycolipid which was included in this work is the acidic form of cellobioselipids. This compound, of which the self-assembly in water has never been reported, is interesting because of its structural similarities to the saturated form of acidic sophorolipids, to which the self-assembly an be compared.

By combining cryo-TEM experiments and pH-resolved in-situ and ex-situ Small Angle X-ray Scattering, we will show that monounsaturated glucolipids, G-C18:1, form vesicles at $\mathrm{pH}<6.2$; saturated glucolipids, G-C18:0, form infinite sheets at $\mathrm{pH}<7.8$; while cellobioselipids, despite their natural and hard-to-avoid mixture of congeners, form chiral 
fibers in large amount at $\mathrm{pH}<7.0$. When comparing these results to the behaviour of sophorolipids, one cannot explain the observed trend using the packing parameter argument alone. Consequently, we believe that the theories of membrane elasticity ${ }^{22,23}$ combined with the physico-chemical environment, and in particular the large difference in the melting temperature, $\mathrm{T}_{\mathrm{M}}$, of oleic acid and stearic acid, regulate the formation of such complex objects. We actually provide the direct proof that this hypothesis is correct by performing combined $\mathrm{pH}$ and temperature-dependent cryo-TEM experiments below $\left(\mathrm{T}=4^{\circ} \mathrm{C}\right)$ and above $\left(\mathrm{T}=80^{\circ} \mathrm{C}\right)$ the $\mathrm{T}_{\mathrm{M}}$ of oleic $\left(13^{\circ} \mathrm{C}\right)$ and stearic $\left(69^{\circ} \mathrm{C}\right)$ acids, the lipidic components of respectively G-C18:1 and G-C18:0.

\section{Materials and Methods}

The chemical structure of the microbial glycolipids used in this work is shown in Figure 1, where glucolipids G-C18:1 and G-C18:0 and hydrolyzed cellobioselipid (CLH) are represented. Glucolipids G-C18:1 and G-C18:0 have been obtained using a modified strain $(\Delta u g t B 1)$ of the yeast Starmerella bombicola. Their chemical structure is respectively equal to the monounsaturated and saturated forms of sophorolipids, ${ }^{26,25}$ except for the presence of a single $\beta$-D-glucose hydrophilic head, instead of a glucose $\beta(1,2)$ disaccharide. CLH is the hydrolyzed acidic derivative of a classical cellobioselipid (CL) mixture produced by the yeast-like strains as Ustilago maydis. ${ }^{31}$ The detailed synthesis and purification procedure is provided here below.

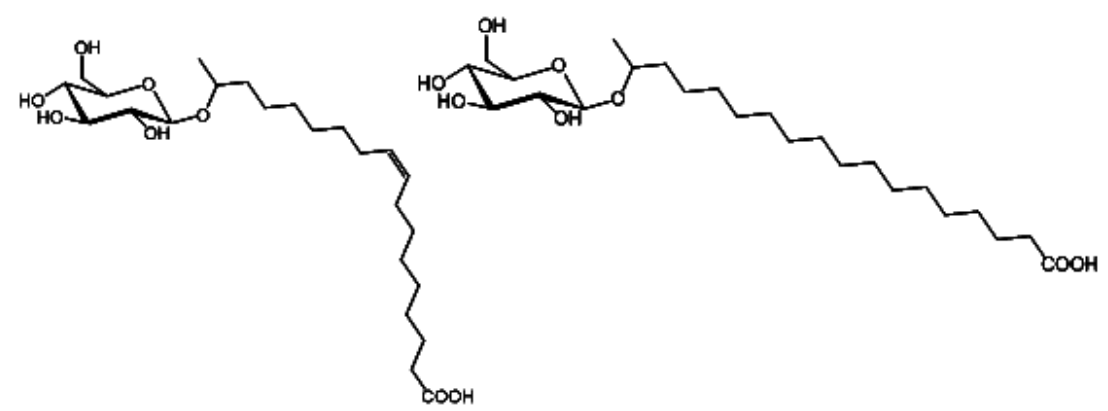

G-C18:1

G-C18:0

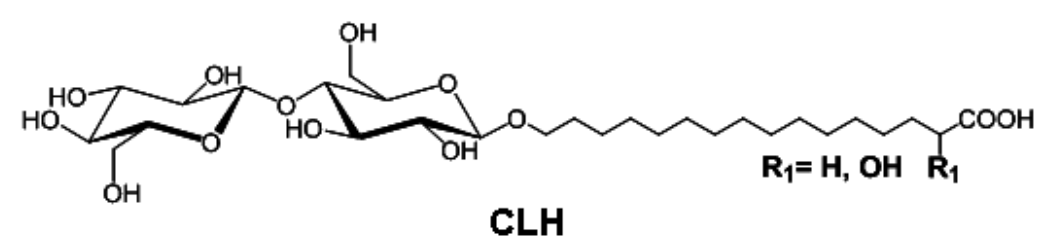

Figure 1 - Chemical formulas of the glycolipids used in this work. G-C18:1, glucolipid bearing a monounsaturated ( $\left.\mathrm{C}_{9,10}\right)$ fatty acid; G-C18:0, glucolipid bearing a saturated fatty acid; $\mathrm{CLH}$, hydrolyzed cellobioselipid mixture 
Synthesis of glucolipid G-C18:1: The S. bombicola strain $\Delta$ ugtB1 described by Saerens et al. ${ }^{32}$ was used to produce glucolipids in a bioreactor experiment. Analytics were done as described by these authors. Pre-cultures were prepared from single colonies in $5 \mathrm{~mL}$ of production medium described by Lang et al. ${ }^{33}$ in round bottomed culture tubes and incubated $\left(30^{\circ} \mathrm{C}, 200\right.$ $\mathrm{rpm}, 30 \mathrm{~h})$. These pre-cultures were inoculated (5\% v/v) in $100 \mathrm{~mL}$ of production medium $\left(30^{\circ} \mathrm{C}, 200 \mathrm{rpm}, 48 \mathrm{~h}\right)$. Two shake flask cultures were used to inoculate the reactor $(20 \% \mathrm{v} / \mathrm{v})$. Cyclic fed-batch fermentations were run in a Biostat ${ }^{\circledR} \mathrm{B}$ culture vessel (Sartorius ${ }^{\circledR}$ ) with a working volume of $1.5 \mathrm{~L}$. The important parameters temperature: $30^{\circ} \mathrm{C}$, stirring: $800 \mathrm{rpm}, \mathrm{pH}$ : 3.5 and aeration: 1 vvm were controlled by the Biostat ${ }^{\circledR}$ B control unit. For maintaining $\mathrm{pH}$ at 3.5, $5 \mathrm{M} \mathrm{NaOH}$ was used. There was no correction for a too alkaline $\mathrm{pH}$ and fermentations started at pH 5.8 and was allowed to drop spontaneously to 3.5. A $500 \mathrm{~g} / \mathrm{L}$ glucose solution was used as a hydrophilic carbon source. Rapeseed oil was fed as hydrophobic carbon source. Glucose was added stepwise in order to have a minimal concentration of $30 \mathrm{~g} / \mathrm{L}$ inside the vessel. Rapeseed oil was added daily (20 to 25 g/day) unless an oil layer appeared after spinning down the cells, then no additional oil was added. Further details of the fermentation process will be described elsewhere. The broth derived from the fermentation was mixed with two volumes of Disolol ${ }^{\circledR}$ ethanol (Chem-Lab). Cells were subsequently removed by centrifugation in a high capacity centrifuge (Thermo Scientific, Sorvall ${ }^{\mathrm{TM}} \mathrm{RC} 6$ Plus) at $4{ }^{\circ} \mathrm{C}$ and $8000 \mathrm{rpm}$ for $15 \mathrm{~min}$. The water-ethanol supernatant was collected and removed by evaporation in a rotavapor (Buchi ${ }^{\circledR}$ ). An HPLC-ELSD chromatogram of the composition of the product is shown in Figure S 1A. An hexane (Fischer Scientific, UK) extraction was performed in order to remove the hydrophobic impurities (oil and fatty acids). This resulted in the formation of three fractions: a water phase containing hydrophilic impurities, a hexane phase containing the oil and free fatty acids and a precipitate consisting of the product (mixture of acetylated and non-acetylated glucolipids).

Hydrolysis of glucolipid G-C18:1: To obtain only non-acetylated glucolipids, alkaline hydrolysis was applied: the product was dissolved in 3 volumes of $5 \mathrm{M} \mathrm{NaOH}$ and the mixture was heated in order to facilitate product dissolution. The solution was subsequently refluxed $\left(20,100^{\circ} \mathrm{C}\right)$ and the $\mathrm{pH}$ was brought back from 13 to 4 by adding $\mathrm{HCl}(36 \%)$. The resulting non-acetylated glucolipids were extracted using tBME. The top organic phase was collected and if present, a middle oily phase was re-extracted. Water fractions containing salt and acetate were discarded, the tBME phases were pooled and the solvent was slowly evaporated under reduced pressure, which resulted in an off white to yellow powder 
consisting of purified non-acetylated glucolipids containing about $90 \%$ of sub-terminal GC18:1 and 7\% of terminal G-C18:1, as one can estimate from the relative peak areas in the HPLC-ELSD presented in Figure $S 1 B$ and Table S1. Additional ${ }^{1} \mathrm{H}$ solution NMR characterization of this compound, presented in Figure S 2, agrees well with the HPLC data. Synthesis of glucolipid G-C18:0: $0.58 \mathrm{~g}$ (1.27 mmol) of deacetylated G-C18:1 glucolipid was dissolved in $50 \mathrm{~mL} \mathrm{MeOH}$ and $0.058 \mathrm{~g}(10 \mathrm{w} / \mathrm{w} \%) \mathrm{Pd} / \mathrm{C}$ was added in portions under $\mathrm{N}_{2}$ atmosphere. The reaction mixture was stirred under 5 bar $\mathrm{H}_{2}$ atmosphere for 7 hours and subsequently filtered over celite. The reaction mixture was concentrated under reduced pressure to yield $0.44 \mathrm{~g}$ (75\%) saturated glucolipid G-C18:0 (Figure 1) as a white powder. This compound, being a derived product of the previously described G-C18:1 batch, was only characterized by ${ }^{1} \mathrm{H}$ solution NMR (Figure S 2), the experimental details of which are given in the Supporting Information.

Synthesis of cellobioselipids (CL): Ustilago maydis DSM $17146^{34}$ was used to produce a mixture of native cellobioselipids. Cells were first cultivated in shaking flasks with $24 \mathrm{~g} / \mathrm{L}$ potato dextrose, at starting $\mathrm{pH}$ of 5.6 for four days in order produce biomass. ${ }^{31,35}$ For CL production cells were harvested, washed with $0.9 \% \mathrm{NaCl}$ and afterwards inoculated with an $\mathrm{OD}_{625 \mathrm{~nm}}=0.8$ and cultivated as resting cells up to 14 days in the $1.7 \mathrm{~g}$ yeast nitrogen base medium (200 mL) with $40 \mathrm{~g} / \mathrm{L}$ saccarose, as pulse wise feed. The cultivation broth was adjusted on $\mathrm{pH} 2.5$ with citrate buffer. After cultivation the broth was centrifuged, the pellet dissolved in ethanol and centrifuged again. The supernatant was separated, evaporated and the CL raw extract was washed with ethylacetate ( $4 \mathrm{~mL} / \mathrm{g}$ CL raw extract) and used for further hydrolysis and investigation. If compared to the glucolipids, this compound is known to be a more complex mixture of different congeners, as described in ref. 31. Such a polydispersity is reproduced in this work, as shown for CL, the LC-MS of which (Figure S 3) shows at least four major components at 15.00, 19.04, 19.35 and 22.92 minutes and which correspond to typical cellobioselipids CL B and CL C reported in ref. 31.

Hydrolysis of cellobioselipids (CLH): to increase the water solubility and to reduce the number of congeners in the CL sample, a hydrolysis step was operated. The as-synthesized $\mathrm{CL}$ have been dissolved in a $1 \mathrm{M} \mathrm{NaOH}$ solution under reflux for 10 minutes at $90^{\circ} \mathrm{C}$. The solution is allowed to reach room temperature and $\mathrm{pH}$ is eventually adjusted below 5 under stirring. A white precipitate forms and can be recovered by either filtration or centrifugation. After washing with milliQ-grade water to remove residual salt and organic acids, the compound is dried and ready to use. As shown by the ${ }^{1} \mathrm{H}$ NMR data in Figure $\mathrm{S} 2 \mathrm{~b}$, this compound can be identified as the hydrolyzed form of CL, referred to as CLH, free of any 
fatty esters and acetyl groups and it is mainly composed of congeners, the difference among which occurs in the hydroxyl chain substitutes. characterization of the $1 \mathrm{H}$ NMR and type of congeners is described in more detail in the Supporting Information. The compound is considered to be free of residual fatty acid and oil and it $s$ used as such without further purification attempted.

Sample preparation: a given amount of the desired glycolipid is dissolved into milliQ-grade water at room temperature to give a concentration of $50 \mathrm{mg} / \mathrm{mL}$ or $5 \mathrm{mg} / \mathrm{mL}$. The $\mathrm{pH}$ is increased up to $\sim 11$ by adding 10-15 $\mu \mathrm{L}$ of $5 \mathrm{M} \mathrm{NaOH}$ solution and it is eventually decreased by adding $\mu \mathrm{L}$ amounts of $0.1 \mathrm{M}-1 \mathrm{M} \mathrm{HCl}$ until the solution becomes turbid. This procedure, necessary to make the glycolipids water-soluble, generates $\mathrm{NaCl}$. However, this is only a minor drawback in the conditions explored as first of all, all systems are prepared in the same way and, consequently, comparable one to the other. Secondly, we have demonstrated that $\mathrm{NaCl}$ has no effect at all on the nature of the self-assembled structure in similar systems, but it only affects aggregation: on micellar-forming monounsaturated sophorolipids, $\mathrm{NaCl}$ only affects the degree of ellipticity of the micelles, ${ }^{28}$ while on chiral-fiber forming saturated sophorolipids, $\mathrm{NaCl}$ only affects fiber aggregation but not their chirality. ${ }^{36}$

Aliquots of the sample are then taken and analyzed by cryo-TEM and SAXS. in-situ experiments (see SAXS section) are performed during the synthesis process while ex-situ experiments, to reduce at best the aging time, are performed within a few hours after synthesis. If needed, samples are cryo-fixed and stored in liquid nitrogen for long period of times (from days to weeks). To prevent temperature bursts between $-196^{\circ} \mathrm{C}$ and room temperature (RT) that could affect the nature of the self-assembled objects, samples are defrosted from $-196^{\circ} \mathrm{C}$ to $\mathrm{RT}$ in the following way: $1 \mathrm{~h}$ at $-80^{\circ} \mathrm{C}$ and $12 \mathrm{~h}$ at $-18^{\circ} \mathrm{C}$, then to room temperature. By this way, we have never observed changes in the type of objects after cryo-fixation.

Transmission Electron Microscopy experiments under cryogenic conditions (cryo-TEM): these experiments were performed on a FEI Tecnai 120 twin microscope operating at $120 \mathrm{kV}$ equipped with a Gatan Orius CCD numeric camera. The sample holder was a Gatan Cryoholder (Gatan 626DH, Gatan). On both microscopes, DigitalMicrograph ${ }^{\mathrm{TM}}$ software was used for image acquisition. Cryofixation was done on a homemade cryo-fixation device. The solutions were deposited on a glow-discharged holey carbon coated TEM copper grid (Quantifoil R2/2, Germany). Excess solution was removed and the grid was immediately plunged into liquid ethane at $-180^{\circ} \mathrm{C}$ before transferring them into liquid nitrogen. All grids were kept at liquid nitrogen temperature throughout all experimentation. Please note that 50 
$\mathrm{mg} / \mathrm{mL}$ samples needed further dilution (generally 10x) for a good cryo-TEM observation. This was performed using the parent solution few minutes before cryo-fixation.

Light Scattering (LS): Light scattering was done on a Zetasizer Nano ZS device using a 633 $\mathrm{nm}$ wavelength and a $90^{\circ}$ geometry. In order to minimize effects of dust and maximize the reproducibility, each sample was analyzed 3 times. Each analysis is characterized by 20 measurements of $5 \mathrm{~s}$ each.

Small Angle X-ray Scattering (SAXS): several publications have shown that the stimuli-driven self-assembly of lipids is a non-equilibrium process; ${ }^{16,17}$ for this reason, we have repeated SAXS experiments on three different instruments using both ex-situ and in-situ analyses, one being complementary to the other. In the ex-situ approach, the self-assembled structures are analyzed within a few hours after their preparation. If this approach leaves more time to reach $\mathrm{pH}$ equilibrium, it does not allow to precisely determine the $\mathrm{pH}$ of the solution aliquot at the moment of analysis. This method is also not compatible with detailed follow-up of the selfassembly process as a function of $\mathrm{pH}$. For this reason, we have developed an in-situ method in which the $\mathrm{pH}$ is controlled and measured at all time during the acquisition of the signal. However, since the time interval between two $\mathrm{pH}$ values is in the order of minutes (details below), this approach does not leave the system too much time to reach equilibrium of $\mathrm{pH}$.

In the ex-situ experiment, the solutions at $\mathrm{pH} \leq 11.6$ are prepared as indicated above. These are sampled and analyzed in a quartz or borosilicate capillary. These experiments have been recorded on a three pinhole type S-MAX 3000 RIGAKU Nanoviewer instrument using a monochromatic $\mathrm{Cu}-\mathrm{K} \alpha$ radiation produced by a microfocus (20 microns x 20 microns) sealed tube X-ray source (MicroMax 002+ RIGAKU working at $40 \mathrm{~W}$ ) and equipped with a twodimensional multi-wire proportional gas detector. The sample-to-detector distance was varied between $1529 \mathrm{~mm}, 1469 \mathrm{~mm}$ and $843 \mathrm{~mm}$. More details are given in the main text. The applied voltage and filament current were $40 \mathrm{kV}$ and $50 \mathrm{~mA}$ respectively. The $q$-range calibration was made using a silver behenate standard sample $\left(\mathrm{d}_{\text {ref }}=58.38 \AA\right.$ ). The measured intensity is always divided by the sample transmission and appropriate masking was done to eliminate the beam-stop shadow contribution and imperfection of the 2D detector. Borosilicate sample holders of $1 \mathrm{~mm}$ diameter have been used. The acquisition time per sample was $1 \mathrm{~h}$.

In addition, SAXS experiments were repeated on freshly-prepared solutions at the SWING beamline of the SOLEIL Synchrotron facility (Saint-Aubin, France): the beam energy was set to $12.6 \mathrm{keV}$ and the high-q region is explored using a sample-to-detector distance of $1 \mathrm{~m}$ while the low-q region is explored using a sample-to-detector distance of $6 \mathrm{~m}$. 
The capillary used to analyze both the background solution (sample-free solution at $\mathrm{pH}$ 11.6) and the samples is never changed, so as to minimize imperfections during data treatment. Samples have been analyzed using an automated robot provided at the beamline and which allows a continuous flow of solution ( $50 \mu \mathrm{L} / \mathrm{min})$ in front of the beam so to reduce possible beam damage. The acquisition time is set between $500 \mathrm{~ms}$ (for low-q) and $300 \mathrm{~ms}$ (high-q). Between 5 and 10 spectra are commonly acquired per sample and averaged, allowing very good statistics on the data. Given the environmental setup, the high-q region for all samples is analyzed within a 30 min timespan after about $4 \mathrm{~h}$ from sample preparation while the lowregion is analyzed after about $7 \mathrm{~h}$.

The in-situ experiments have employed a flow-through polycarbonate $2 \mathrm{~mm}$ capillary connected to the sample-containing solution at $\mathrm{pH} 11.6$ through a peristaltic pump. The $\mathrm{pH}$ was controlled in-situ via a classical $\mathrm{KCl} \mathrm{pH}$-meter directly in the experimental hutch and constantly monitored. $\mathrm{pH}$ changes have been obtained by using a $0.1 \mathrm{M} \mathrm{HCl}$ solution introduced via a motor-controlled press-syringe. If the flow-through device reduces beam damage, we could only acquire 1 spectrum at each $\mathrm{pH}$ under this particular set-up, thus making the statistics more fluctuating than in the ex-situ experimental conditions. Data have been acquired on the high brilliance ID02 beamline at the ESRF synchrotron (Grenoble, France). Error-bars on the ID02 experiments were calculated based on the estimated number of photons detected (accounting for the gain and quantum efficiency of the CCD and phosphor layer), assuming a Poisson statistics. The noise of the detector was accounted for by comparison of darks.

In all cases, data have been acquired using a CCD camera and integrated azimuthally to obtain a typical I(q) spectrum. Contribution of the solvent (water at $\mathrm{pH} 11.6$ ) and capillary have been measured prior to the experiment and duly substracted during the data treatment. All SAXS data (in-situ and ex-situ setups) have been corrected for the transmission of the direct beam and scaled to be in absolute scale.

Nuclear Magnetic Resonance (NMR): solution ${ }^{1} \mathrm{H}$ NMR data presented in Figure S 2 have been acquired on a Bruker $300 \mathrm{MHz}$ liquid spectrometer using a ${ }^{1} \mathrm{H}-\mathrm{X}$ BBFO probe. Classical one-pulse experiments using a recycling delay of $1 \mathrm{~s}$ have been used to record data while 2D COSY45 and TOCSY (delay= $80 \mathrm{~ms}$ during spin-lock using a MLEV-17 sequence) ${ }^{37}$ experiments (Figure S 4) have been used to determine the structure of CLH. The 3.2 version of the TOPSPIN software was used for acquisition and treatment of data. Standard pulse programs found in the Bruker-TOPSPIN pulse programme library have been used.

pH titration was done on a solution containing $5 \mathrm{mg} / \mathrm{mL}$ of a given compound in water, the 
solution $\mathrm{pH}$ of which is initially settled at $\mathrm{pH} \sim 11.6$ using $10-15 \mu \mathrm{L}$ of $5 \mathrm{M} \mathrm{NaOH}$ solution. Titration is done using $\mu \mathrm{M}$ amounts $(\sim 2-5 \mu \mathrm{L})$ of a $0.5 \mathrm{M} \mathrm{HCl}$ solution.

Circular Dichroism (CD) has been recorded on a Jasco J-810 spectropolarimeter between 190 $\mathrm{nm}$ and $600 \mathrm{~nm}$ with a $0.1 \mathrm{~nm}$ step for solutions at a concentration of $50 \mathrm{mg} / \mathrm{mL}$ of the given sample solution. The compound is initially dissolved at $\mathrm{pH} \sim 11.6$ in deionzed water and $\mathrm{pH}$ was successively decreased with $0.5 \mathrm{M} \mathrm{HCl}$ solutions and then loaded into a $1 \mathrm{~mm}$ quartz cuvette for measurements.

HPLC-ELSD analysis. HPLC-ELSD analysis was performed with the Agilent 1260 Infinity equipped with a Agilent Zorbax Eclipse Plus C18 column (4.6 x 100 mm - 3.5 micron) at $40^{\circ} \mathrm{C}$. A flow rate of $1 \mathrm{~mL} / \mathrm{min}$ was applied and a gradient of two solvents (A: 0,05 \% acetic acid and B: acetonitrile) was applied using the following method: 0 min: 95\% A and 5\% B, 25 min: 5\% A and 95\% B, 27 min: 5\% A and 95\%B and 30 min: 95\% A and 5\% B.

LC-MS analysis: analysis of CL was done with Thermo LCQ Deca by the use of RP18-solid phase (150x 2 mm, Phenomenex Luna) and solvents (i) methanol with 0.1 formic acid and (ii) water with $0.1 \%$ formic acid as gradients. LC-MS analysis of glucolipids was done on a a Shimadzu LC-10-AD HPLC system (Shimadzu Europe GmbH, Germany) connected to a quadrupole mass spectrometer (Waters, Milford, MA). Molecules were identified by their native molecular masses after ESI (electron spray ionization) without collision.

\section{Results}

The products that have been used in this study are all obtained from microbial synthesis, a process which brings a natural diversity in terms of molecular structure. Glucolipid G-C18:1 contains no detectable amount of residual free fatty acids or vegetable oil ( $<0.005$ and $<0.01 \%$ respectively). $90 \%$ of the glucolipids correspond to the structure depicted in Figure 1: the fatty acid moiety is composed of an oleyl chain hydroxylated at the subterminal position, $7 \%$ of the molecules harbour the same oleyl being terminal hydroxylated, so $97 \%$ of the sample corresponds to glucolipids with a C18:1 fatty acid chain. The remainder of the mixture is composed of molecules carrying C18:2 and C18:0 fatty acid chains. Upon hydrogenation, all double bonds are saturated resulting in a nearly $100 \%$ pure G-C18:0 sample. CLH is a more heterogeneous mixture. The full ${ }^{1} \mathrm{H}$ NMR analysis of the compounds is provided in the Supporting Information. All compounds revealed to be poorly soluble in water at room temperature. For this reason, and to explore their assembly behaviour, the same procedure followed in ref. 25 was adopted. A given amount of the compound is dissolved in water by increasing the $\mathrm{pH}$ of the solution to $\mathrm{pH}>11$, then, $\mathrm{HCl}$ is 
added to decrease the $\mathrm{pH}$. This operation can be followed both by $\mathrm{pH}$ titration and light scattering, which is used to probe the turbidity of the solution. Figure 2a shows the evolution of the scattered intensity as a function of $\mathrm{pH}$. For all systems, the count rate at $\mathrm{pH}$ above 9 is constant, the absolute value of which varies between each compound. The non-zero values of the scattered intensity indicates that the ionic form of all compounds undergoes self-assembly in water, even if at this stage it is impossible to determine the nature and the amount of such aggregates. At pH between 6 and 8, a strong increase in the scattered intensity is recorded for all compounds, a clear sign of massive solution turbidity, as also visible by the naked eye. In the acidic $\mathrm{pH}$ range, the value of scattered light is generally high and stays practically constant. However, in some cases (e.g., CLH, G-C18:1), abrupt decrease in intensity at $\mathrm{pH}<5$ can occur; since the solution is still highly turbid, the abrupt decrease most likely depend on phase separation between the precipitate and water. Even if the above behaviour is common to all compounds, each one has its own characteristics. The sharp clear/turbid transition for GC18:1 and G-C18:0 respectively occurs at $\mathrm{pH}=6.2 \pm 0.3$ and $7.8 \pm 0.4$, while the same phenomenon for CLH seems to occur in two $\mathrm{pH}$ steps. At $\mathrm{pH}=7.0 \pm 0.4$, one can observe a small increase in intensity from about $100 \mathrm{kcps}$ to about $500 \mathrm{kcps}$, while at $\mathrm{pH}=6.2 \pm 0.4 \mathrm{a}$ very sharp increase is eventually recorded. A clearer view of this double behaviour is given in the combined $\mathrm{pH} /$ intensity evolution with an amount of $\mathrm{HCl}$ in solution (expressed in the form of total concentration in the final solution). Figure $2 d$ shows that for the CLH system the large drop of $\mathrm{pH}$ from 11 to about 7, corresponding to the titration of the $\mathrm{NaOH}$, has no influence on the scattered intensity. However, at higher amounts of $\mathrm{HCl}$, the $\mathrm{pH}$ is practically constant at the value of 7 , while the scattering intensity gently increases up to about $700 \pm 100$ kcps. It is observed that the equilibration time per $\mathrm{pH}$ value requires several minutes. Protonation of the $\mathrm{COOH}$ group, and formation of self-assembled objects, is more likely to start at this stage. Finally, as soon as the $\mathrm{pH}$ drops again, the scattered intensity undergoes a sharp increase, a phenomenon which could also be related to morphological changes. In comparison, G-C18:1 and G-C18:0 have a more straightforward behaviour, as the scattered intensity undergoes a single transition in the $\mathrm{pH}$ region where a pseudo-plateau is observed (Figure 2b,c). 

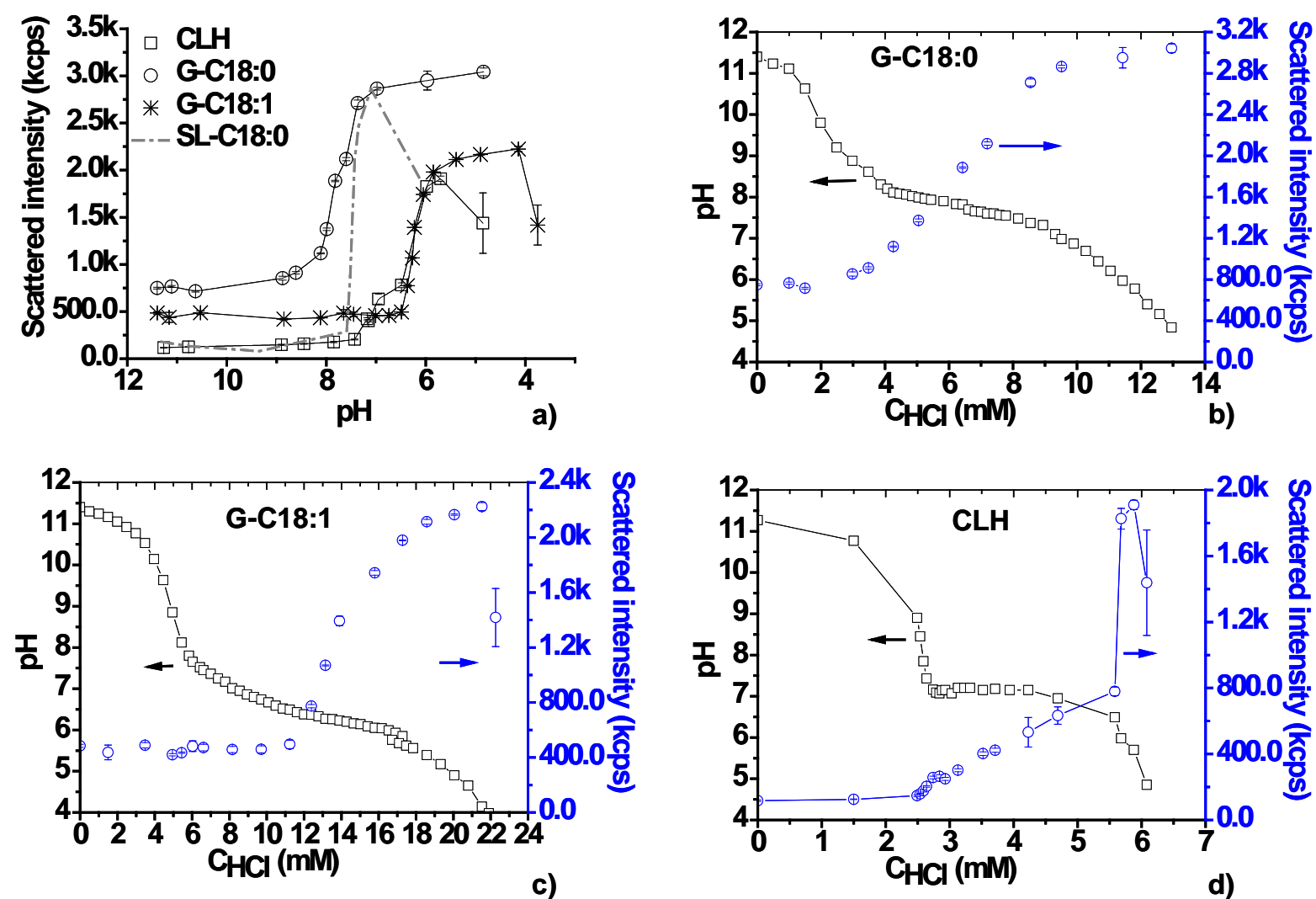

Figure 2 - a) Turbidity measurements as a function of pH for G-C18:1, G-C18:0, CLH glycolipids. Data for the saturated acidic sophorolipid compound, SL-C18:0, has been adapted from Ref. 25. b-d) Titration and turbidity measurements experiments combined for (b) G-C18:0, (c) G-C18:1 and (d) CLH

The $\mathrm{pH}$-driven turbidity evolution of these compounds can be directly compared to the one measured on sophorolipids. ${ }^{25}$ Sophorolipids containing a cis- double bond do not form any remarkable large scale structures upon $\mathrm{pH}$ decrease in the neutral/acidic region. However, fully saturated sophorolipids (SL-C18:0) show the sudden formation of twisted ribbons at about $\mathrm{pH}=7.4 \pm 0.2$. For convenience, the $\mathrm{pH}$-driven scattered light transition for SL-C18:0 is reported on Figure 2a (segmented-dot line). The evidence that each compound has its own well-defined transition $\mathrm{pH}$, not necessarily in the same range (a difference of $2 \mathrm{pH}$ units is recorded between G-C18:0 and G-C18:1) is unexpected, and its possible origin will be commented later after a more detailed description of the morphologies both at basic and acidic pH media.

\section{Basic $p H$}

Figure 3 shows the cryo-TEM and SAXS results for the CLH, G-C18:0 and G-C18:1 compounds at $\mathrm{pH}$ 11, at which light scattering (Figure 2a) and SAXS (Figure S 5) profiles are 
unchanged over a pH range between 11.5 and 9.5. When looking at $\mathrm{CLH}$, the grid is covered with large cloudy aggregates (Figure 3a) constituted of ill-defined filamentous matter (Figure 3b,c), similarly to what is found for the SL C18:0 compound in the same pH range. ${ }^{29}$ However, and differently from the latter, the CD signal of the CLH (Figure S 6) at basic pH seems to be different from zero, thus indicating the presence of chiral self-assembled objects, even if this cannot be visualized directly by the cryo-TEM images, as they not resolved enough. The typical in-situ SAXS at pH 11 for this sample (Figure 3d) has one main contribution in the low-q region, characterized by a slope value of $-1.45 \pm 0.04$. Surprisingly, a broad signal above $\mathrm{q}>0.1 \AA^{-1}$ seems to indicate that CLH in the basic medium does not form well-defined micelles, as was found for instance in SL-C18:1 and SL-C18:0 sophorolipids at equivalent $\mathrm{pH} .{ }^{29}$ This seems to suggest the coexistence of the supramolecular assemblies with surfactant monomers, a fact which, even if unexpected, is not uncommon, as described by Bryskhe et al., ${ }^{17}$ who found that the lamellar phase of a non-ionic surfactant system obtained from the temperature treatment of a starting micellar solution actually coexists with a dilute solution of the surfactant monomers only.

The G-C18:0 sample is characterized by a much better defined in-situ SAXS signature (Figure 3h): the low-q region has a $-2.05 \pm 0.04$ slope, typical for flat morphologies, while the high-q region ( $\mathrm{q}>0.04 \AA^{-1}$ ) shows the typical signature of micellar objects. The value of the minimum of the first oscillation, attributed to the form factor of a micelle, ${ }^{29}$ is $q_{\min }=0.17 \AA^{-1}$. In the case of a homogeneous sphere, the minimum of the form factor can be used to estimate the radius of the sphere according to the classical equation $\mathrm{Rq}_{\min }=4.49$; if, in this case, the estimated value is $\mathrm{R}=26.4 \AA$, one must take this value with caution due to the nonhomogeneous nature of glycolipid micelles. ${ }^{28}$ The cryo-TEM images presented in Figure 3e-g seem to be in agreement with SAXS: they show the presence of vesicles, which co-exist with tubular structures, while the background is constituted of smaller micellar aggregates. Interestingly, these objects even seem to interact with each other, as shown in Figure 3g, in which vesicles are contained in hollow tubes, or in Figure 3f, showing two, or more, hollow tubes into the other. Finally, the G-C18:1 sample appears to form a complex network of filamentous aggregates (Figure 3i,k), a fact which is also corroborated by the $-1.28 \pm 0.05$ slope at $\mathrm{q}<0.02 \AA^{-1}$ in the in-situ SAXS experiment (Figure 3l), which does not exclude the presence of micelles, as shown by the typical scattering profile at $q>0.02 \AA^{-1}$. The minimum of the first oscillation $\mathrm{q}_{\min }=0.19 \AA^{-1}$, similar to what it found for the G-C18:0 compound, indicating micelles close in size between these two glucolipids. 

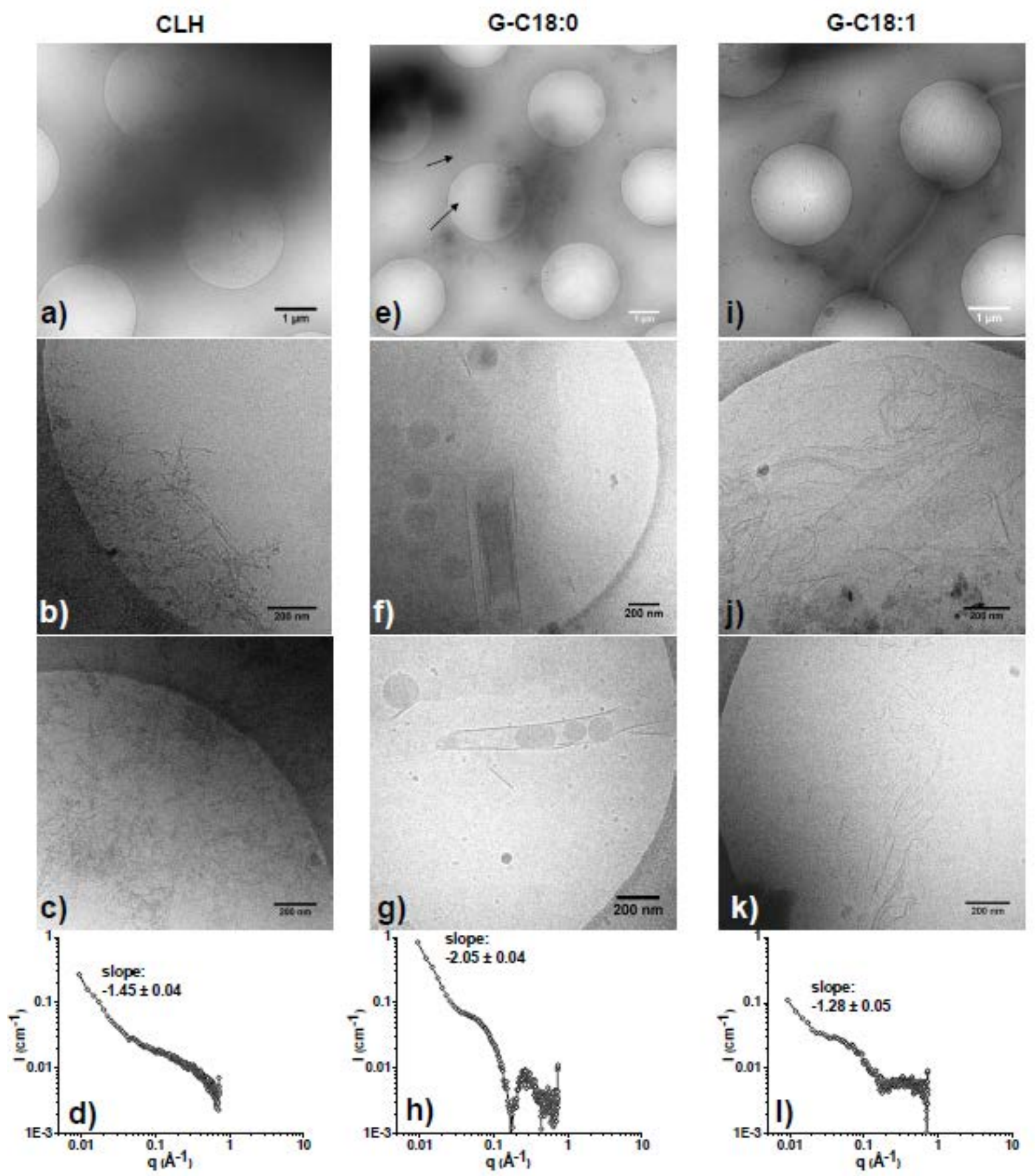

Figure 3 - CLH : (a-c) cryo-TEM images and (d) in-situ SAXS. G-C18:0: (e-g) cryo-TEM images and (h) in-situ SAXS. G-C18:0: (i-k) cryo-TEM images and (l) in-situ SAXS. All experiments have been performed on $5 \mathrm{mg} / \mathrm{mL}$ solutions at $\mathrm{pH} \sim 11$. Small Angle $\mathrm{X}$-ray Scattering experiments have been recorded on the ID02 line of ESRF synchrotron (Grenoble, France).

Acidic $p H$

I. Glucolipid G-C18:0 - Formation of infinite bilayers

The cryo-TEM analysis of the G-C18:0 compound in the $\mathrm{pH} 7.8 \pm 0.4$ region is shown in Figure 4. At low magnification (Figure 4a), one can observe the presence of infinitely long 
superimposed flat sheets. Arrows 1 and 2 respectively indicate one sheet layer and the junction between two superimposed sheets. At higher magnification, in Figure 4b, one can identify the presence of several sheets, the borders of which are highlighted by arrows 3 and 4; arrow 5 indicates the junction between two sheets. Finally, at higher magnification (Figure 4c), one can have the detail of the sheet border: a high electron density region indicated by arrow 6 and a low electron density region indicated by arrow 7. These clearly indicate that the sheets borders are folded at the extremities, undoubtedly to reduce line tension, ${ }^{19}$ but open ends can occasionally occur (arrow 7). Interestingly, additional, small, objects can be detected in the proximity of the sheets, as shown by arrow 8 .
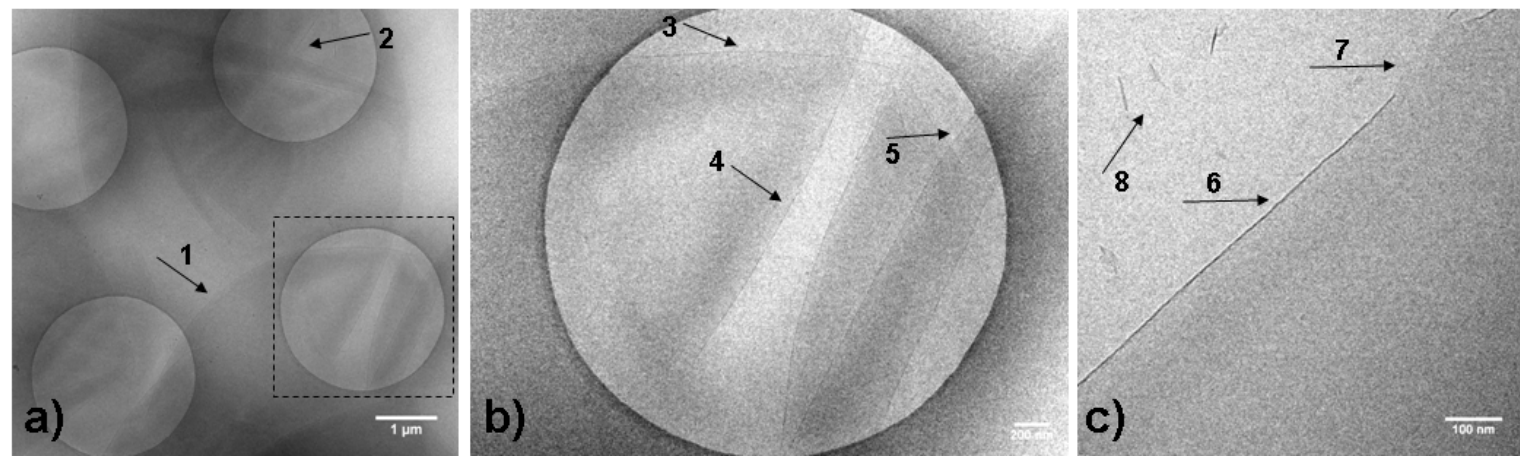

Figure 4 - Cryo-TEM images of the infinite self-assembled sheets formed with G-C18:0 at pH $\sim 7.8$

These can often be found next to the sheets borders, as also shown by Figure 5a. Upon tilting the sample holder (Figure 5b), the acicular 1 and 2 objects tend to disappear while at location 3 one can see an acicular object appearing. This is characteristic of a flat disk morphology (diameter estimated between $80 \mathrm{~nm}$ and $100 \mathrm{~nm}$ ), the electron density contrast of which is very low, hence undetectable, when their flat surface is orthogonal to the incident electron beam. It is possible that these sparse objects are related, or even precursors, to the presence of the infinite flat sheets, as this was described in phospholipid/cholate salt mixtures. ${ }^{19}$ The CD experiments in Figure S 6 show a flat, zero, signal throughout the spectrum, thus confirming the fact that no chiral self-assembled object is formed. 


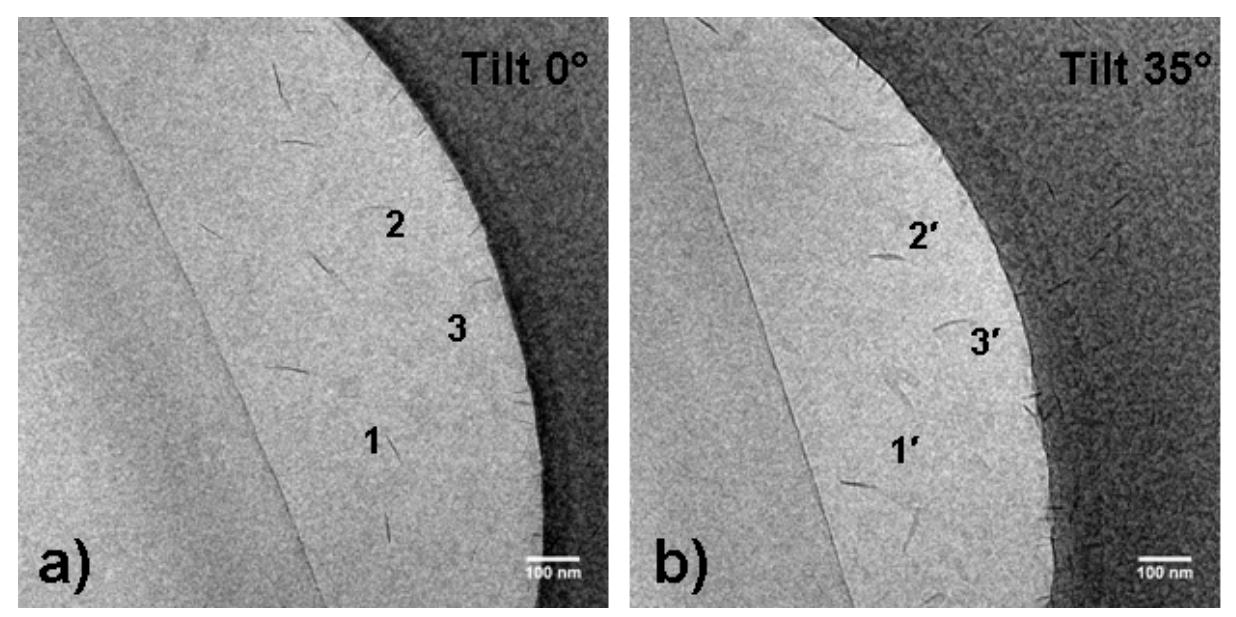

Figure 5 - High-magnification cryo-TEM images recorded for the G-C18:0 sample at pH 7.8 at two tilt angles a) $0^{\circ}$ and b) $35^{\circ}$ of the sample holder

The in-situ SAXS data of G-C18:0 (Figure 6a) have been recorded in the middle of the transition region at pH 7.54 and below pH 5. First of all, one can observe an intense scattering signal at low-q values with a slope of $-2.2 \pm 0.1$, a value which is agreement with the presence of flat objects. The fact that a plateau is not reached at $0.01 \AA^{-1}$ is also in agreement with the presence of structures at least larger than $63 \mathrm{~nm}$. The signal at high q values renders the oscillation of a core-shell flat morphology, the form factor of which can be employed to model the entire SAXS curve (Figure S 7) and for which the core-shell model is necessary to account for the broad oscillation. The position of the minimum of the oscillation of the SAXS profile is informative on the half section of a lamellae according to the relationship $\mathrm{qR}=\pi$ valid for a homogeneous object. Here, the position of the minimum is $0.12 \AA^{-1}$, so one can estimate half the section to about $26 \AA$, a value which, as commented before, must be taken as indicative due to the core-shell nature of the bilayer. However, given the fact that the size of G-C18:0 could reasonably be estimated to be less than $30 \AA$, using the Tanford formula to estimate the stearic acid moiety ( $\leq 22 \AA$, see legend of Table 2 for more details) and estimating the size of $\beta$-D-glucose to be less than $8 \AA{ }^{38}$ one can still make the reasonable hypothesis that the membrane is constituted by a double layer of G-C18:0, even if it is not excluded that interdigitation between the top and bottom layers actually occurs. Differently from the CLH case, the SAXS profile above $0.12 \AA^{-1}$ does not contain diffraction peaks, but it is characterized by the oscillation due to the form factor of the lamellae alone. This supports the idea that the glucolipid molecules are organized perpendicularly (tilting is not excluded) to the membrane surface, as classically found in lipid bilayers. However, the diffraction peak at $0.16 \AA^{-1}$ at $\mathrm{pH}=3.29$ clearly indicates a (pseudo-)crystalline molecular packing in the plane of the membrane. It is interesting to note that the SAXS profile does not change between $\mathrm{pH}$ 
7.54 and $\mathrm{pH} 4.93$, suggesting little or no morphological evolution in this $\mathrm{pH}$ range. However, at lower, more acidic values, a solid precipitate with lamellar molecular packing is observed, as indicated by the first and second order diffraction peaks at $0.16 \AA^{-1}$ (39.3 $\AA$ ) and $0.32 \AA^{-1}$ (19.6 A). Given the multicomposite nature of these peaks one can argue that the lamellar structure coexists with the flat bilayer sheets.

As the in-situ experiments are very useful to follow up the morphological evolution at any $\mathrm{pH}$ value, the fast rate of $\mathrm{pH}$ change is known to potentially influence the self-assembly process, known to be kinetically-driven for similar systems. ${ }^{16,17,19}$ The ex-situ SAXS spectra are presented in Figure 6b. First of all, one can recognize the similar scattering profile previously observed in-situ, indicating that the mode of preparation does not have a striking influence on the overall morphology. In particular, the SAXS spectrum recorded below $\mathrm{pH} 4$ shows the same diffraction pattern at $\mathrm{q}>0.16 \AA^{-1}$. Nevertheless, an extra broad diffraction peak is detected in the low-q portion of the spectrum at about $0.025 \AA^{-1}$ at $\mathrm{pH} 4$. The same peak at $0.022 \AA^{-1}$ can also be documented at higher concentration in the $\mathrm{pH} 6$ environment. This broad peak identifies a repeating distance of about $260 \AA$, attributed here to an average inter-sheet repeating distance, most likely due to the spontaneous interactions of the sheets. This hypothesis is supported by the fact that the G-C18:0 basic solution becomes highly viscous, and eventually jelly, after introduction of the acid and one needs to vigorously stir it to make it more fluid. This is very important in the in-situ experiment, in which, if the stirring is not strong enough, one can experience the clogging of the probe capillary. As it can be seen in the cryo-TEM image in Figure 4a, the sheets tend to aggregate and pile one on top of each other, a phenomenon which seems to be enhanced in the static, ex-situ, experiment, thus providing an inter-sheet diffraction signal. The lack of a clear inter-sheet diffraction peak in the in-situ experiments seems to indicate that the stirring step is vigorous enough to beak apart the "infinite" sheets into smaller ones. 

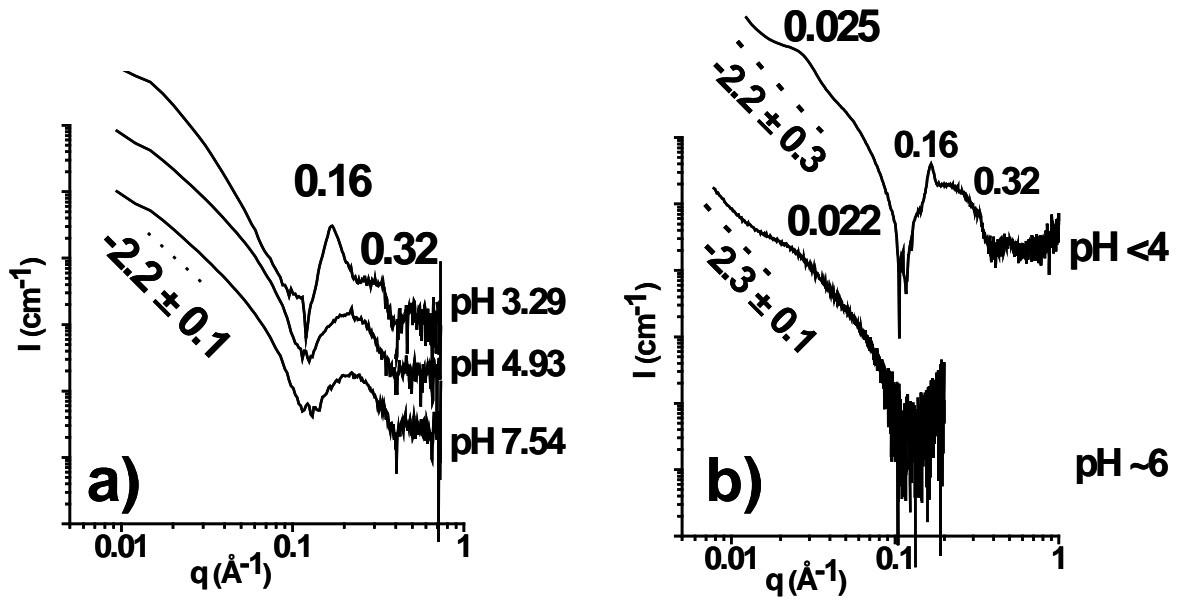

Figure 6 - a) pH-resolved in-situ (ID02 line, ESRF synchrotron, Grenoble, France) and b) ex-situ SAXS experiments recorded on the G-C18:0 sample. Pattern at $\mathrm{pH}<4$ has been recorded on the SWING line, SOLEIL synchrotron, Saint-Aubin, France. Pattern at $\mathrm{pH} \sim 6$ has been recorded on a lab-scale SAXS instrument with a sample-to-detector distance of $1469 \mathrm{~mm}$

\section{Glucolipid G-C18:1- Formation of vesicles}

The G-C18:1 sample has a similar structure as G-C18:0 except for the presence of a monounsaturated cis $\mathrm{C}=\mathrm{C}$ bond at positions 9,10 in the hydrocarbon chain. Its clear-turbid transition $\mathrm{pH}$ is settled in the vicinity of $\mathrm{pH}$ 6.2, as shown by the light scattering experiments. The cryo-TEM images (Figure 7) recorded in this $\mathrm{pH}$ range show an interesting mixture of two types of objects. Figure 7b shows the presence of vesicles, the cross diameter of which ranges from tens to a hundred of nanometers, or even more. Figure 7c shows the existence of entangled giant micelles, the junctions of which are pointed at by black arrows. Interestingly, we could also find aggregates showing that large vesicular objects merge with the giant micelles (Figure 7a,d). 

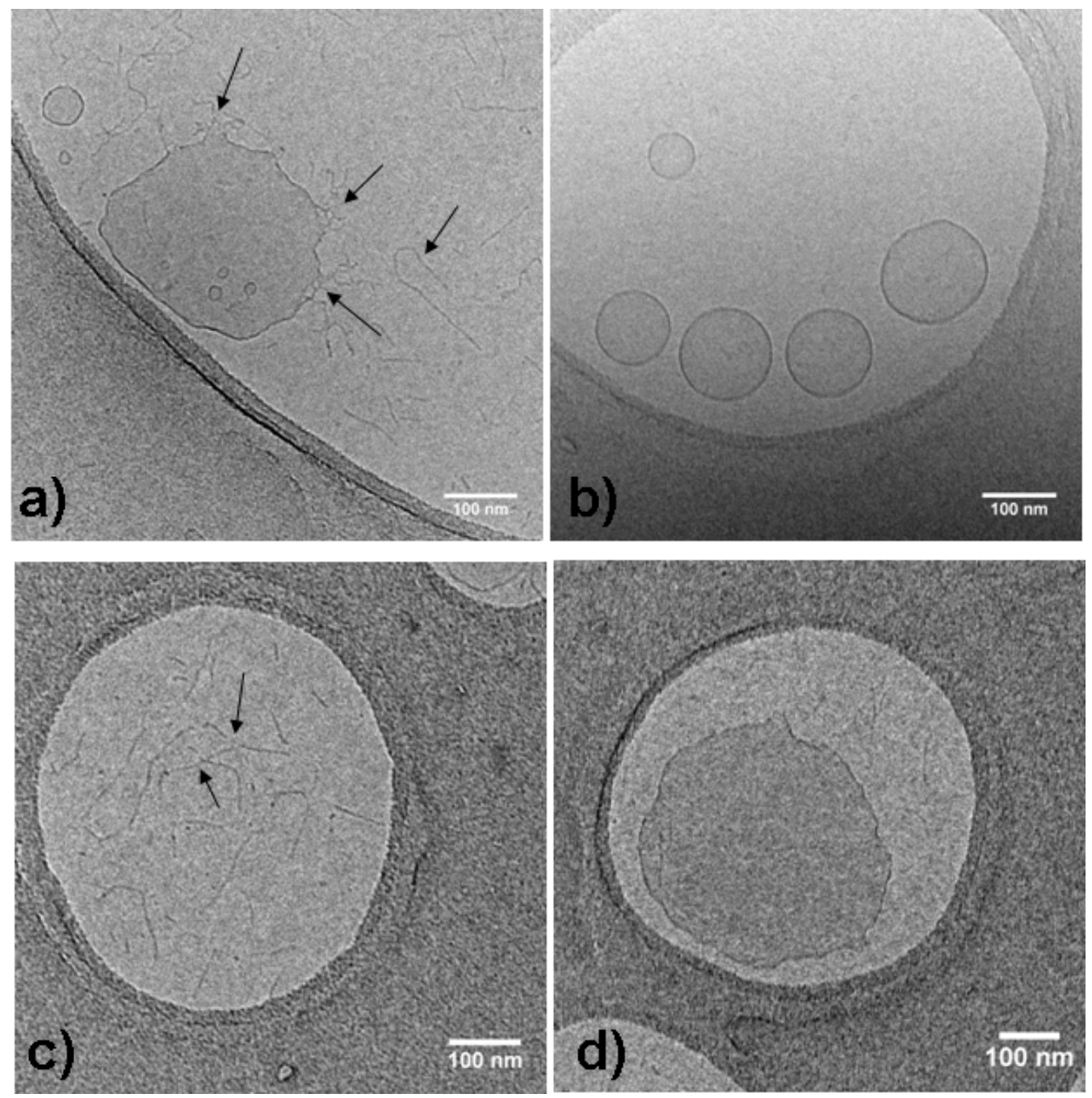

Figure 7 - Cryo-TEM images of the a) giant micelles/vesicles, b) vesicles, c-d) giant micelles and giant vesicles formed with G-C18:1 at $\mathrm{pH} \sim 6.2$

Since the cryo-TEM images in Figure 7 are taken in a transition state of the sample, we have carried out the same experiment at $\mathrm{pH}<6$, just below the transition point. This is shown in Figure 8, where both uni- and multi-lamellar vesicles are found, with no trace of giant micelles. This shows that the $\mathrm{pH}$ behaviour of this compound involves a high-curvature cylindrical intermediate (at least) in the neutral $\mathrm{pH}$ range and evolving towards low-curvature vesicular structures at lower $\mathrm{pH}$. The CD experiments in Figure S 6 show a practically null signal at acidic $\mathrm{pH}$, confirming the fact that no chiral self-assembled object is formed on a large scale. 

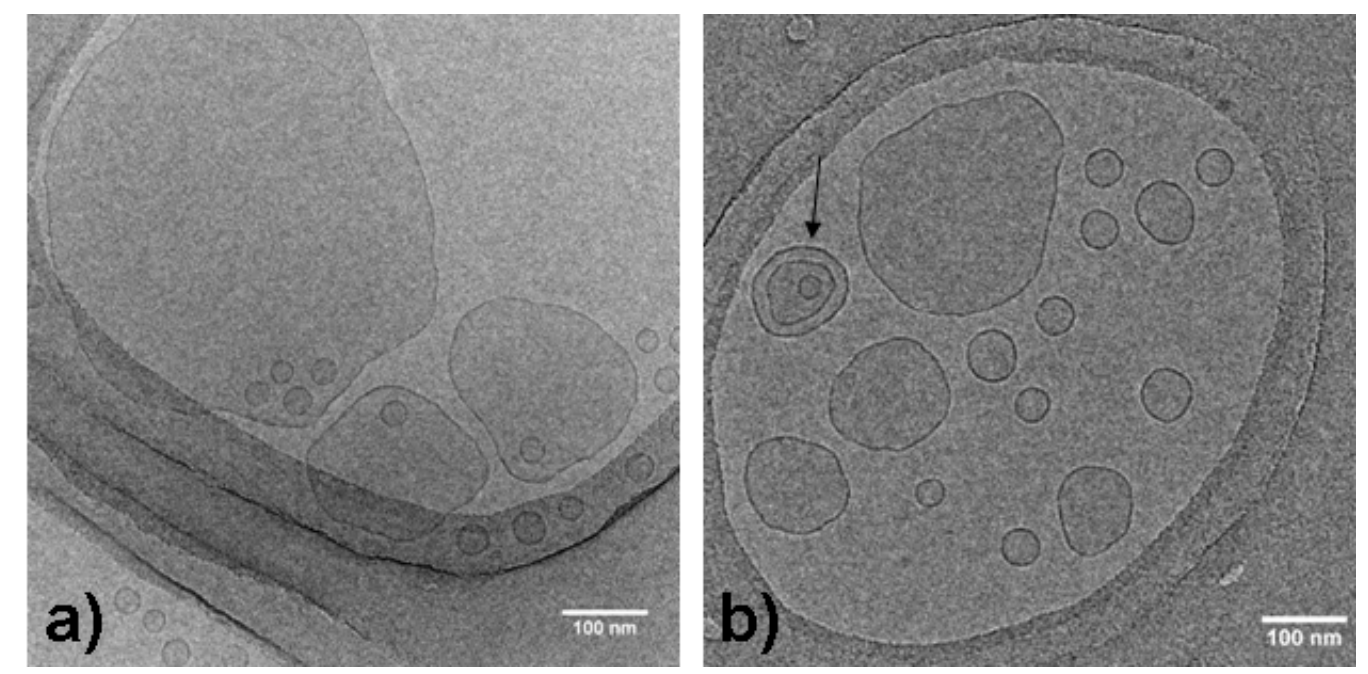

Figure 8 - Cryo-TEM images of the vesicles formed with G-C18:1 at pH $<6$

This trend can nicely be followed by pH-driven in-situ SAXS experiments shown in Figure 9a. The SAXS pattern recorded at a $\mathrm{pH}$ just above the transition ( $\mathrm{pH}$ 6.62) shows the typical feature of cylindrical objects, as suggested by the monotonous increase in the scattering intensity at low q with a $-1.27 \pm 0.02$ slope. Upon decreasing the $\mathrm{pH}$ and approaching the transition in the vicinity of $\mathrm{pH}$ 6.2, one can see slight but significant variations in both the low-q slope and form factor oscillation above $\mathrm{q}>0.1 \AA^{-1}$. The q-portion between $0.1 \AA^{-1}$ and $0.01 \AA^{-1}$ can now be divided into a $-2.29 \pm 0.03$ and a $-1.23 \pm 0.21$ slope region, respectively characteristic of flat interfaces and tubular objects. The simultaneous existence of both regimes is in very good agreement with the cryo-TEM images given in Figure 7. According to the minimum position of the oscillation $\left(0.12 \AA^{-1}\right)$ one can estimate a half of the bilayer membrane thickness to be about $26 \AA$, in agreement with the estimated molecular size of GC18:1 ( $<30 \AA$ ) and with what it found in the G-C18:0 system. Interestingly, the position of the minimum of the oscillation does not vary between the micellar morphology at $\mathrm{pH}=6.62$ and the vesicular shape at $\mathrm{pH}<6.60$; this proves that the vesicle membrane bilayer forms directly from the tubular morphology, as shown in Figure 7a. At $\mathrm{pH}<4$, one can find the typical diffraction pattern $\left(0.18 \AA^{-1}\right.$ and $\left.0.36 \AA^{-1}\right)$ of a lamellar packing superimposed to the vesicle signal, indicating that a lamellar precipitate (visible by the naked eye) coexists with the vesicles.

The ex-situ SAXS experiments, presented in Figure 9b, show similar features if compared to the in-situ data. Nevertheless, one main difference should be highlighted, especially at higher concentrations: a broad diffraction peak observed in the $0.04<\mathrm{q}\left[\AA^{-1}\right]<$ 0.05 region. This peak identifies a repeating distance in the range of $140 \AA$, which we attribute to an average inter-vesicle distance measured in multilamellar vesicular systems, as 
in the one indicated by the arrow in Figure 8b. We do observe, as for the stacking of sheets in the G-C18:0 glucolipid, that an ex-situ preparation of the sample allows the formation of multi-walled vesicles, which does not seem to appear in the in-situ preparation of the sample. These minor discrepancies seem to be coherent with the kinetics-driven self-assembly behaviour expected in pH-jump systems and described earlier for other compounds. ${ }^{16,19}$
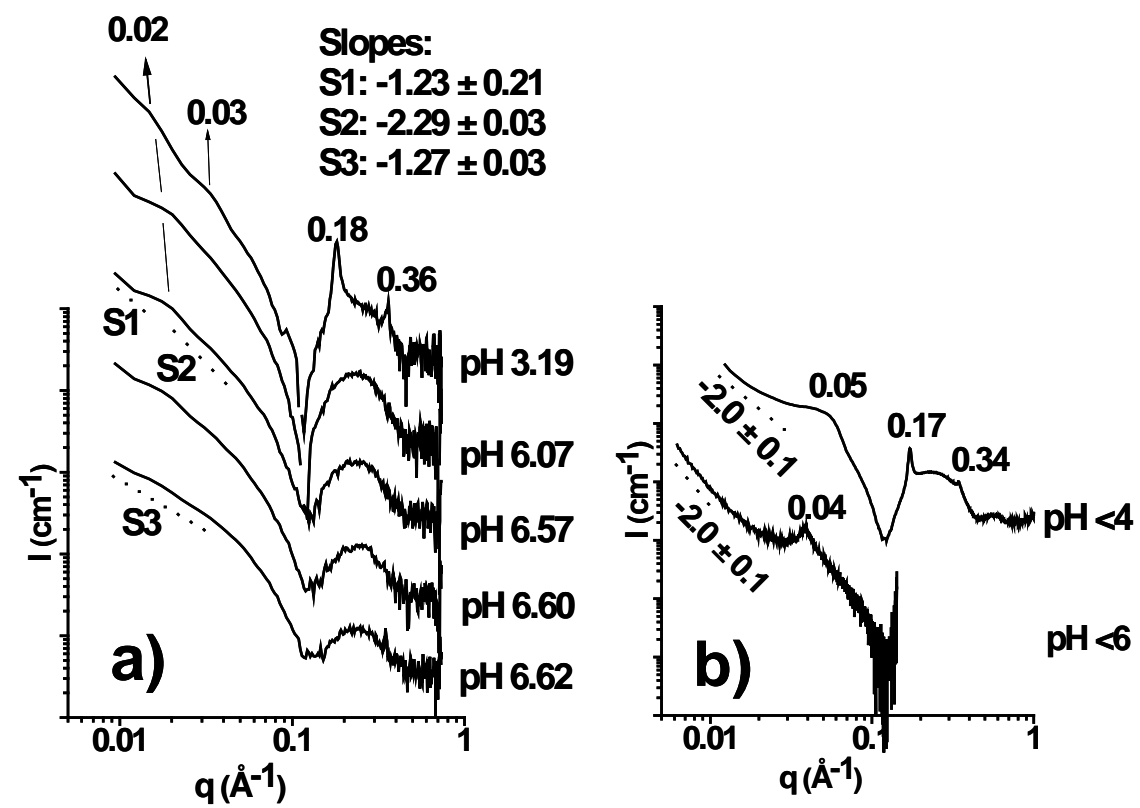

Figure 9 - a) pH-resolved in-situ (ID02 line, ESRF synchrotron, Grenoble, France) and b) ex-situ SAXS experiments recorded on the G-C18:1 sample. Pattern at $\mathrm{pH}<4$ has been recorded on the SWING line, SOLEIL synchrotron, Saint-Aubin, France. Pattern at $\mathrm{pH}<6$ has been recorded on a lab-scale SAXS instrument with a sample-to-detector distance of $1529 \mathrm{~mm}$

III. The case of cellobioselipids - formation of crystalline fibers

According to LS experiments in Figure 2, CLH displays two pH regions with different light scattering behaviour. Figure 10 shows the cryo-TEM images recorded on the CLH system at $\mathrm{pH}<7$. In particular, Figure 10a-d refer to the initial transition $\mathrm{pH}$ range $(7.0 \pm 0.4)$, where the solution becomes turbid, while Figure 10e-h refer to the $\mathrm{pH}$ range below 7 , where a strong intensity uptake in the LS experiments was found. Figure 10a shows a typical overview of the CLH sample, showing the presence of an entangled network of elongated fibers. A closer look within one of the amorphous ice layer holes (Figure 10b,c) shows the presence of at least two types of objects indicated by the numbered arrows: twisted fibers $\left(\mathrm{N}^{\circ} 1,4\right)$ and helical fibers $\left(\mathrm{N}^{\circ} 2,5\right)$. The presence of vesicular objects of about $25 \mathrm{~nm}$ in diameter (Figure 10d) is not excluded but caution should be taken to interpret these objects as real because 
typical pollution in cryo-TEM experiments could also explain them. For this reason they will not be commented further. Arrow $\mathrm{N}^{\circ} 3$ points at an object which could be interpreted as a flat ribbons or hollow nanotubes; the latter are excluded because the electron density contrast is homogeneous throughout the fiber section. Circular dichroism experiments (Figure S 6) confirm the presence of helical/twisted self-assembled structures at acidic $\mathrm{pH}$. In a similar system, constituted by the saturated form of sophorolipids, ${ }^{25}$ a compound that can be compared both in terms of size and shape to CLH, twisted ribbons were found to be the main type of fibrillar morphology under similar physico-chemical conditions.

Table 1 - Typical morphologies, cross-section and pitch for the main fibrillar objects found in the cryoTEM images of CLH samples at acidic pH. Values are given in $\mathbf{n m}$.

\begin{tabular}{|c|c|c|}
\hline Morphology & $\begin{array}{c}\text { Cross- } \\
\text { section/diameter }\end{array}$ & Pitch \\
\hline Helical fiber & $5.5 \pm 0.5$ & $60 \pm 1$ \\
\hline Flat ribbon & $6.0 \pm 0.5$ & - \\
\hline $\begin{array}{c}\text { Twisted } \\
\text { fibers }\end{array}$ & $11.0 \pm 1.0$ & $110 \pm 2$ \\
\hline
\end{tabular}
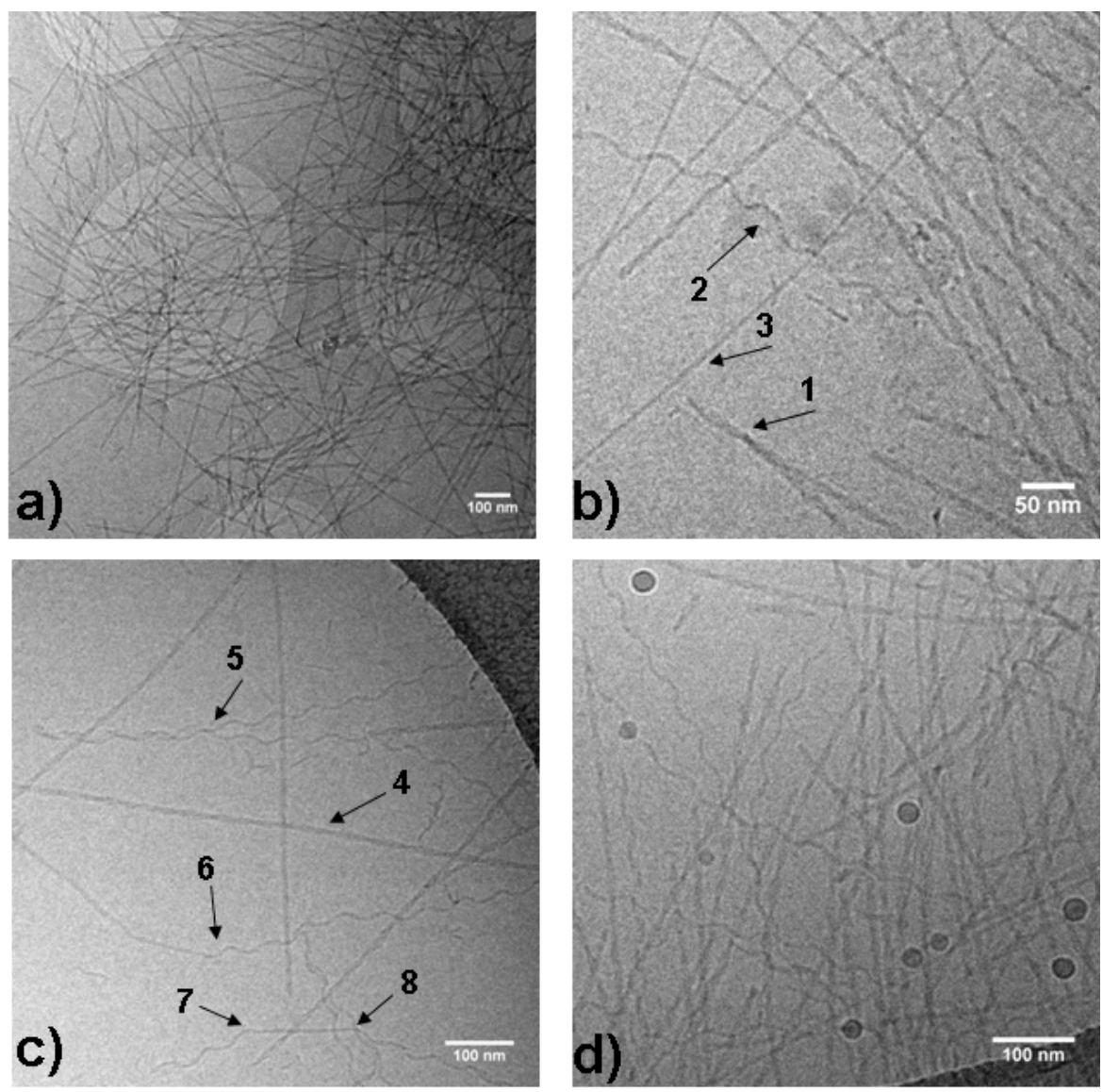

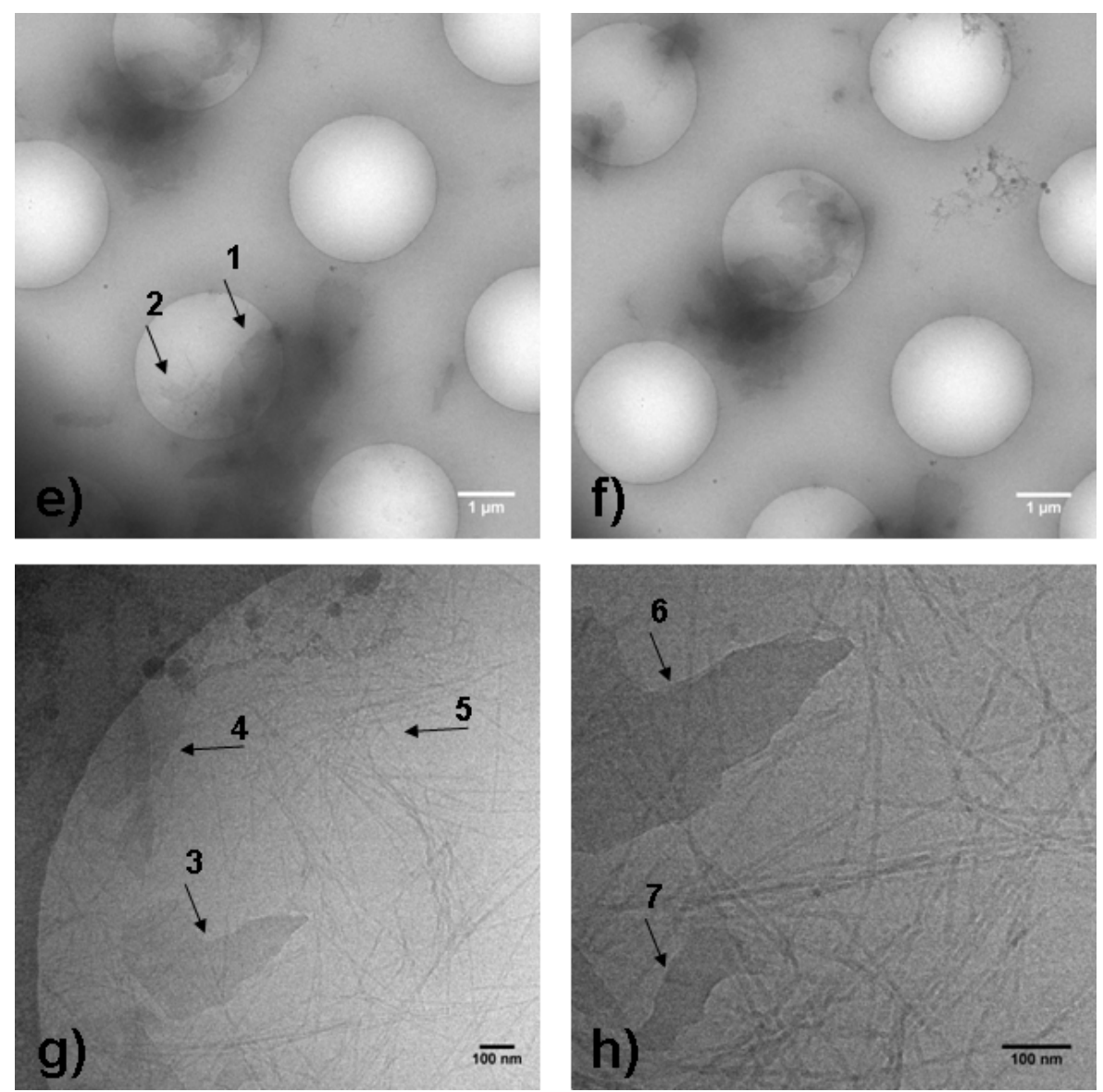

Figure 10 - Cryo-TEM images of self-assembled aggregates formed with CLH at (a-d) pH $\sim 7$ and (e-h) pH < 5.5. All experiments refer to a sample at $50 \mathrm{mg} / \mathrm{mL}$. To record good quality and representative images, the initial solution was diluted immediately before cryo-fixation of a factor 1/10 (even 1/100 was also tested).

In Table 1 we provide the typical diameters and pitch size for each morphology. If one could attribute the morphology dispersion to the CLH mixture of congeners (see Supporting Information for more details), we believe that they are precise structural connections between them, as described for other systems. ${ }^{39}$ Arrows $\mathrm{N}^{\circ} 6-8$ in Figure 10c point at specific breaking points in which a clear helical/flat ribbon transition occurs. The left-hand side of arrow $\mathrm{N}^{\circ} 6$ identifies a flat ribbon while its right-hand side shows a helical fiber. Similarly, a flat ribbon region is identifiable in between arrows 7 and 8, the exterior part of which turn into helical fibers. The highlighted arrows 7-8 in Figure 10c suggest that helical fibers and flat ribbons are actually part of the same object at two different growth stages.

Twisted ribbons seem to constitute a minority in terms of population and they are at least double the size of the fibers (cross-section of 5-6 nm). It is known that twisted ribbons are precursors for helical fibers, ${ }^{40}$ which generally constitute the last morphological stage before hollow nanotube formation. However, since we observe a direct flat/helical transition, 
it is possible that twisted ribbons in the CLH system are actually not morphologically connected to the helical fibers.

Upon reducing the $\mathrm{pH}$ value below 5.5, at which a strong increase in the scattered light is recorded in light scattering experiments, cryo-TEM shows the formation of large platelet aggregates (Figure 10 e,f), still in coexistence with the fibers (Figure 10g,h), indicating that a morphological transition is taking place. The transition can be followed by $\mathrm{pH}$-resolved insitu Small Angle X-ray Scattering, which show a broad diffraction peak at $0.17 \AA^{-1}$ above $\mathrm{pH}$ 5 , attributed to interplanar order within the ribbons, and a series of sharp diffraction peaks below $\mathrm{pH}$ 5, attributed to the crystalline order within the flat sheets. SAXS data are presented and discussed in detail in Figure S 8.

\section{Discussion}

The pH-dependent behaviour of CLH, G-C18:1 and G-C18:0 are very similar to what was previously found for the saturated form of acidic sophorolipids ${ }^{25}$ and other glycolipids. ${ }^{21,41}$ Nevertheless, the transition between the high $\mathrm{pH}$, low-scattering, to the low$\mathrm{pH}$, high-scattering, regimes occurs at sensibly different $\mathrm{pH}$ values among the compounds. If this fact could seem odd because of the strong resemblance of the fatty acid tails for all compounds, one should not forget that saturated and unsaturated (poly or mono) fatty acids do not necessarily have the same pKa. For instance, the apparent pKa values of a palmitic/oleic acid mixture vary between 6.2 (10\% oleic) and 7.3 (90\% oleic) ${ }^{42}$ and it is known that a 2-unit pKa change occurs between stearic acid and linolenic acid. ${ }^{43}$ It was even shown that the apparent pKa of oleic acid in a mixture with glycerol monooleate varies between 6 (22\% oleic acid) and 7 (80\% oleic acid). ${ }^{44}$ The light scattering experiments presented in this work seem to show that the transparent/turbid transitions are correlated with the pKa trend of the corresponding fatty acids: the compound containing stearic acid as backbone (G-C18:0 and SL-C18:0) undergo a transition at a pH higher than the compound containing oleic acid (GC18:1), a fact which is coherent with the higher pKa values measured for stearic compared to oleic acid. ${ }^{43}$ Finally, CLH, mainly composed of a partially-hydroxylated hexadecanoic acid, shows its main transition at pH 6.2. According to Ref. 43, 45, the pKa of hexadecanoic acid is two units smaller than stearic acid and in Ref. 42 the apparent pKa of a 90:10 palmitic acid (= hexadecanoic acid)-oleic acid mixture was estimated to be 6.2.

Table 2 compares the estimated packing parameters for sophorolipids, glucolipids and cellobioselipid. One should recall that the packing parameter, $p$, was proposed to correlate the 
molecular geometry of an amphiphile to the type of supramolecular aggregate this forms above its critical micellar concetration. ${ }^{20} p$ is defined as the ratio between the volume of the aliphatic chain, $V$, and the product between the surface area occupied by the polar head, $a_{0}$, and the chain length, $L: p=\frac{V}{a_{0} L}$. Even if an exact value of $p$ is difficult to estimate for the glycolipids in this study, one can safely state that acidic sophorolipids bearing an oleic acid moiety, SL-C18:1, have $p=0.36$, close to the upper limit value $(0.3)$ for the spherical/cylindrical micellar transition. $V$ and $L$ are estimated using the well-known Tanford formulas in which we considered 16 effective $\mathrm{CH}_{2}$ groups in the aliphatic chain $\left(\mathrm{C} 18\left(\mathrm{CH}_{3}\right)\right.$ and $\mathrm{C} 17(\mathrm{CH})$ positions are not included). The value of $a_{0}$ is more complicated to determine. We have used an effective $75 \AA^{2} /$ molecule ${ }^{28}$ for the sophorolipid/water interface, this value being estimated at the dry core/hydrated shell interface and in agreement with previous works on disaccharides amphiphiles. ${ }^{46}$ We have also considered that the $120^{\circ}$ angle of the oleic acid moiety reduces its effective length to about $16.9 \AA$, opposed to $21.8 \AA$ calculated for a fully extended 16-atoms aliphatic chain. The estimation for this compound is in very good agreement with the experimental results, because SL-C18:1 forms ellipsoidal micelles in the acidic $\mathrm{pH}$ range at room temperature, the packing parameter of which is expected to be slightly above 0.3 .

Table 2 - Calculated and experimental packing parameter for the glycolipids studied in this work.

* Intended under the same conditions: $\mathrm{pH}<6,0.5 \mathrm{w} \%, \mathrm{~T}=25^{\circ} \mathrm{C}$. $\S$ The value of the packing parameter is estimated as follows: the reference $a_{0}$ value is taken as the surface per sophorolipid at the dry core/hydrated shell interface and it is estimated to be $75 \AA^{2}$, an average experimental value determined in Ref. 28 for the SL-C18:1 system. These same assumptions have been applied to the CLH system. The $a_{0}$ for the glucolipids samples is simply considered to be half the value of the sophorolipid compounds. $L$ and $V$ are calculated with the classical Tanford formula, $L=1.54+1.265^{*} n$ and $V=27.4+26.9 n$, where $n$ is the number of carbon atoms, here taken as 16 for all compounds (the C17 and C18 positions are considered to be part of the hydrophilic headgroup) except for $\mathrm{CLH}$, for which we used the value of 14 (COOH and $\mathrm{C} 2$ in $\alpha$ are excluded given the presence of an $\mathrm{OH}$ group in the latter). For the C18:1 compounds, bearing a $120^{\circ} \mathrm{C}=\mathrm{C}$ double bond located at $\mathrm{C}_{9,10}, \mathrm{~L}$ is estimated to be as the $\mathrm{C10}-\mathrm{C} 16$ portion of the molecule plus the projection of the $\mathrm{C} 1(\mathrm{COOH})$ to $\mathrm{C} 9$ segment, calculated as $\mathrm{L}_{1-9} * \cos (60)$. $(\mathrm{L}=16.9 \AA)$

\begin{tabular}{|c|c|c|c|c|}
\hline Sample & Morphology* $^{*}$ & $\begin{array}{c}\text { Calculated } \\
\text { packing } \\
\text { parameter }^{\S}\end{array}$ & $\begin{array}{c}\text { Experimental } \\
\text { packing } \\
\text { parameter }\end{array}$ & Ref \\
\hline SL-C18:1 & $\begin{array}{c}\text { Ellipsoidal } \\
\text { micelles }\end{array}$ & 0.36 & $\sim 0.3$ & 27 \\
\hline
\end{tabular}




\begin{tabular}{|c|c|c|c|c|}
\hline G-C18:1 & Vesicles & 0.72 & $0.5-1.0$ & This work \\
\hline G-C18:0 & Infinite sheets & 0.56 & 1.0 & This work \\
\hline SL-C18:0 & $\begin{array}{c}\text { Twisted ribbons } \\
\text { Flat sheets }\end{array}$ & 0.28 & 1.0 & 25 \\
\hline CLH & $\begin{array}{c}\text { Twisted and } \\
\text { helical ribbons }\end{array}$ & 0.28 & 1.0 & This work \\
\hline
\end{tabular}

Similar good agreement can be found for the vesicle-forming monounsaturated G-C18:1 glucolipid, the calculated $p$ for which is comprised between 0.5 and 1.0. However, one can immediately realize that such a good agreement between experimental data and calculation has not a general trend; in fact, the values of $p$ estimated for all other samples practically fall below 0.6 , whereas the nature of the self-assembled objects could rather be explained by $p=1$. $p$ alone cannot explain at all the formation of infinite sheets for the G-C18:0 sample. The formation of twisted ribbons for SL-C18:0, the $p$-value of which is unitary, is even more puzzling: this compound has a sophorose head, bulkier than the glucose head in G-C18:0 and for this reason one would expect the formation of low curvature objects, which is not the case. In fact, the main partial conclusion is that it is not possible to predict the self-assembly of these microbial glycolipids on simple molecular geometrical considerations; other factors must be taken into account.

The extensive literature describing the self-assembly properties of surfactants commonly evokes the problem of membrane curvature and flexibility ${ }^{22}$ related to the melting point of the lipid moiety. ${ }^{16,21}$ The giant micelles-to-vesicle transition observed in the G-C18:1 sample is undoubtedly explained by the presence of the less bulky glucose headgroup, with respect to the sophorose disaccharide. The fact that this compound has the same oleic acid moiety as SL-C18:1 is a good hint of the fact that the headgroup plays the most important role in driving the formation of micelles, or vesicles. Oleic acid has a melting point, $\mathrm{T}_{\mathrm{M}}$, of $13^{\circ} \mathrm{C}$ while stearic acid has a $\mathrm{T}_{M}$ of $69^{\circ} \mathrm{C}$. According to this very large $\mathrm{T}_{M}$ difference, the chain fluidity in C18:1-based compounds at RT $\left(25^{\circ} \mathrm{C}>\mathrm{T}_{\mathrm{M}}\right)$ is higher than in C18:0-based compounds, which are expected to form a tighter intermolecular packing and, consequently, more rigid membranes. ${ }^{47}$ If one could have not predicted the formation of an infinite sheet by the G-C18:0 sample, one can nevertheless explain it by the fact that this compound, being very similar in chemical formula and geometry to the vesicle-forming G-C18:1, is actually a C18:0 derivative, expected to form similar, but more rigid, lipid bilayers. The fact that the intermolecular packing plays such an important role is also demonstrated by the fact that a rigid, crystalline, layer is also obtained even with an additional glucose molecule: the 
sophorose-containing SL-C18:0 compound forms flat ribbons. ${ }^{25}$ It is interesting to note that the role of the second glucose molecule does not play in favour of higher curvature morphologies, probably because it is unable to break the tight crystalline packing. However, it seems to have a symmetry-breaking role which destabilizes the formation of the planar layer by blocking its infinite growth and driving the formation towards unidirectional twisted ribbons. ${ }^{40}$ This hypothesis could be supported by the fact that CLH also forms chiral fibers. If this compound has a similar structure as SL-C18:0, it does not share the same chiral center on the the C17 atom of the fatty acid backbone, thus indicating that the fiber chirality may not be related to molecular chirality. Additionally, since sophorose is a $\beta 1,2$-glucose while cellobiose is a $\beta 1,4$-glucose, it means that the chiral fiber formation process is also probably not configuration-dependent, as one could suppose from previous literature works. ${ }^{8}$

Finally, it is also possible that the chiral fibers are a metastable state in the formation of nanotubes, ${ }^{39,48}$ even if this was not demonstrated so far on these systems. One should not forget that the structures studied here are probably not the thermodynamical ones, as shown in various studies on related compounds and where the rate at which a so-called "pH-jump" or “temperature-jump" could be used to control the morphology of self-assembled lipids. ${ }^{16,17}$ This aspect could actually explain the reason why the same SL-C18:0 sophorolipids can form either flat sheets, ${ }^{24}$ if simply mixed with water at acidic $\mathrm{pH}$, or twisted ribbons, ${ }^{25}$ if they undergo a "pH-jump” process. In either case, the experimental packing parameter is unitary, thus demonstrating once again the inconsistency of a simple approach based on molecular topology.

If the considerations above can partially explain the consistency among our data, they cannot explain the difference in morphology between the G-C18:0 sample, forming infinite bilayer membranes, and a very similar compound extensively studied by Masuda et al. ${ }^{30}$ forming nanotubes. The major difference between the two molecules resides in the chemical nature of the linker between the glucopyranoside ring and the fatty acid: acetal group in GC18:0 and a carbamoyl group in the case of Masuda's sample. The latter introduces lateral intermolecular H-bonding interactions, which apparently have a strong effect on the system’s mean curvature, which is zero for infinite sheets and positive for nanotubes, where the mean curvature is defined by $\langle\mathrm{H}\rangle=0.5\left(\mathrm{c}_{1}+\mathrm{C}_{2}\right)$, with $\mathrm{c}_{1}$ and $\mathrm{c}_{2}$ being the principal curvatures defined at a given point of the surface, and $c_{1}=1 / R_{1}$ and $c_{2}=1 / R_{2}$, with $R_{1}$ and $R_{2}$ the radii of curvature. For a plane, $\mathrm{c}_{1}=\mathrm{c}_{2}=0$, hence $<\mathrm{H}>=0$; for a tube, $\mathrm{c}_{1}=0$ and $\mathrm{c}_{2}>0$, hence $<\mathrm{H}>>0$. Interestingly, the carbamoyl group, instead of strengthening the stability of the sheet in its planar form, seems to destabilize it only in one direction belonging to the plane of the 
curvature $c_{2}$. This effect can also be seen as a unidirectional decrease in the packing parameter due to an apparent increase in the hydrophilic surface area. Whatever the case, these behaviours would have been very hard to forecast.

The phenomenological description above is not uncommon for amphiphiles and similar behaviours are described for many phospholipid-based systems, by far the most studied compounds, but also for non-ionic compounds and glycolipids. In ethyleneglycolbased surfactants it was shown that vesicle formation is favoured over the formation of a lamellar phase by increasing the temperature, ${ }^{17}$ while the temperature-dependent evolution of membrane curvature for these systems was discussed already in $1994 .{ }^{23}$ However, it was also shown that size evolution of phospholipid membranes can occur at a given temperature by simply varying the amount of unsaturation of the lipid moiety: large vesicles (small curvature) are observed with increasing number of double bonds; this phenomenon corresponds to reduce the lipids $T_{M}$, a fact which is equivalent to increasing the temperature for a given $\mathrm{T}_{\mathrm{M}} .{ }^{16}$ and references therein Other stimuli-responsive morphological evolutions include $\mathrm{pH}$-driven shape variation, as discussed by Klijn et al. ${ }^{21}$ for carbohydrate-based gemini surfactants. Their work demonstrates that at low $\mathrm{pH}$ values, when the surfactant polar head is positively charged (ammonium salt headgroup), the membrane curvature is high and the system forms giant micelles. Interestingly, the length of the spacer (up to a certain limit), the size of the carbohydrate or the stereochemistry have little influence on the final morphology in this regime. According to them, the positively-charged polar head has a much stronger influence on the local principal curvature than other parts of the molecule. Upon an increase in $\mathrm{pH}$, and neutralization of the polar headgroup charge, they observe a micelle-to-vesicle transition or even inverse micelles, having $p>1$ with a strong impact of the molecular geometry. These results are consistent with our own findings on both SL-C18:1 and SL-C18:029 but also GC18:1 and G-C18:0 compounds in this work. Upon increasing the $\mathrm{pH}$, the carboxylic acid deprotonates thus introducing a negative charge at one end of the glycolipid molecule, the effect of which is to drive the system towards the formation of micelles and nanoscale platelets for SL-C18:1 and SL-C18:0;29 micelles are also observed for the G-C18:1 and GC18:0 compounds, according to the SAXS signal above $q>0.02 \AA^{-1}$ presented in Figure 3h,l. These pieces of evidence support the idea that the negative charge dominates the effective surface area, thus sensibly lowering the packing parameter, responsible for the formation of high-curvature objects. Interestingly, when the $\mathrm{pH}$ is lowered for the G-C18:1 sample, we observe a typical micelle-giant micelles-vesicle transition, which has largely been described for glycolipid, ${ }^{21}$ phospholipid/detergent mixtures, ${ }^{49,50}$ and ethylene glycol surfactants, ${ }^{17}$ 
among others. Leng et al. ${ }^{19}$ have recently settled the theoretical ground for vesicle formation in a typical lecithin/bile salt system; they evoke kinetics arguments, rather than thermodynamics, as the main driving force in the micelle-to-vesicle transition and, in particular, for the growth mechanisms of micelles into disks and their stability after growth by fusion. However, the fact that these morphologies are non-equilibrium intermediates make this system very parameter-dependent (ionic force, temperature, $\mathrm{pH}$, mixing order, time rate, etc...) and, for this reason, very hard to study and to fully understand. If the self-assembly of the selected microbial glycolipids at $\mathrm{pH}<7$ was explained here above on arguments based on their rationalized molecular structures, the more peculiar $\mathrm{pH}$-driven self-assembly behaviour will be described elsewhere.

To support our hypothesis according to which membrane fluidity of G-C18:1composed vesicles and G-C18:0-composed sheets is temperature-dependent, we have repeated the self-assembly process for these compounds at temperatures respectively below $\left(\mathrm{T}=4^{\circ} \mathrm{C}\right)$ and above $\left(\mathrm{T}=80^{\circ} \mathrm{C}\right)$ the $\mathrm{T}_{\mathrm{M}}$ of oleic $\left(\mathrm{T}_{\mathrm{M}}=13^{\circ} \mathrm{C}\right)$ and stearic $\left(\mathrm{T}_{\mathrm{M}}=69^{\circ} \mathrm{C}\right)$ acids, the lipidic components of G-C18:1 and G-C18:0. Experimentally, the initial glycolipid basic solutions at $\mathrm{pH}>11$ are either cooled or heated and $\mathrm{pH}$ is then adjusted to about 5.5 , that is below the corresponding transition points depicted in Figure 2. In a first approximation, we neglect the temperature effect on the exact transition $\mathrm{pH}$ and we analyze solutions which become evidently turbid. After $\mathrm{pH}$ adjustment, the final solutions are stored at either $5^{\circ} \mathrm{C}$ or $80^{\circ} \mathrm{C}$ during $24 \mathrm{~h}$ until analysis and they are never brought to room temperature. The corresponding cryo-TEM images of G-C18:1 at $\mathrm{T}=5^{\circ} \mathrm{C}$ and $\mathrm{G}-\mathrm{C} 18: 0$ at $\mathrm{T}=80^{\circ} \mathrm{C}$ both at $\mathrm{pH}$ about 5.5 are displayed, respectively, in Figure 11 and Figure 12. In the G-C18:1 system below the $\mathrm{T}_{\mathrm{M}}$ of oleic acid, vesicles typically omnipresent at room temperature are more hardly found while large flat sheets (Figure 11a) or curved extended surfaces (Figure 11b) can be observed instead (additional images are given in Figure S 9). On the contrary, when GC18:0 is synthesized at $\mathrm{T}=80^{\circ} \mathrm{C}$, one can find a coexistence between infinitely long flat sheets and vesicles (arrows $\mathrm{N}^{\circ} 1$ and 2 in Figure 12a and Figure S 10a,b). Interestingly, Figure $12 \mathrm{~b}$ shows the presence of a surface the outer edges of which are segmented (arrows $\mathrm{N}^{\circ} 3$ ), most likely indicating the mechanism by which the infinitely long flat sheet of zero mean curvature evolve towards more curved vesicular surfaces. Additional images collected on the same sample show similar effects (Figure S 10d), including the perpendicular stacking between flat sheet. The upper left-hand side of Figure S 10c clearly shows the bending process of a flat sheet around an already-formed vesicle and about to form, presumably, a single multi lamellar vesicle, as already observed in Figure S 10b. 
These experiments directly prove the joint importance of $\mathrm{pH}$ and temperature in the selfassembly experiments for this set of microbial glycolipids, strengthening the fact simple assumptions based on their shape alone can neither predict nor explain the typical selfassembled objects formed at room temperature and that one must reason out these systems also on the basis of the relative $\mathrm{T}_{\mathrm{M}}$ of the lipid part of molecule. These results are schematized on Figure 13, showing the transition $\mathrm{pH}\left(\mathrm{pH}_{\mathrm{T}}\right)$ values between the micelle and the vesicle/bilayer regions for monounsaturated and saturated glucolipids at ambient temperature $\left(T_{a}\right)$, respectively higher and lower than their $T_{m}$. The effect of lowering $T$ below and above $\mathrm{T}_{\mathrm{m}}$ is also indicated. The $\mathrm{pH}$-dependent behaviour of cellobioselipids at $\mathrm{T}_{\mathrm{a}}$ is also represented. 

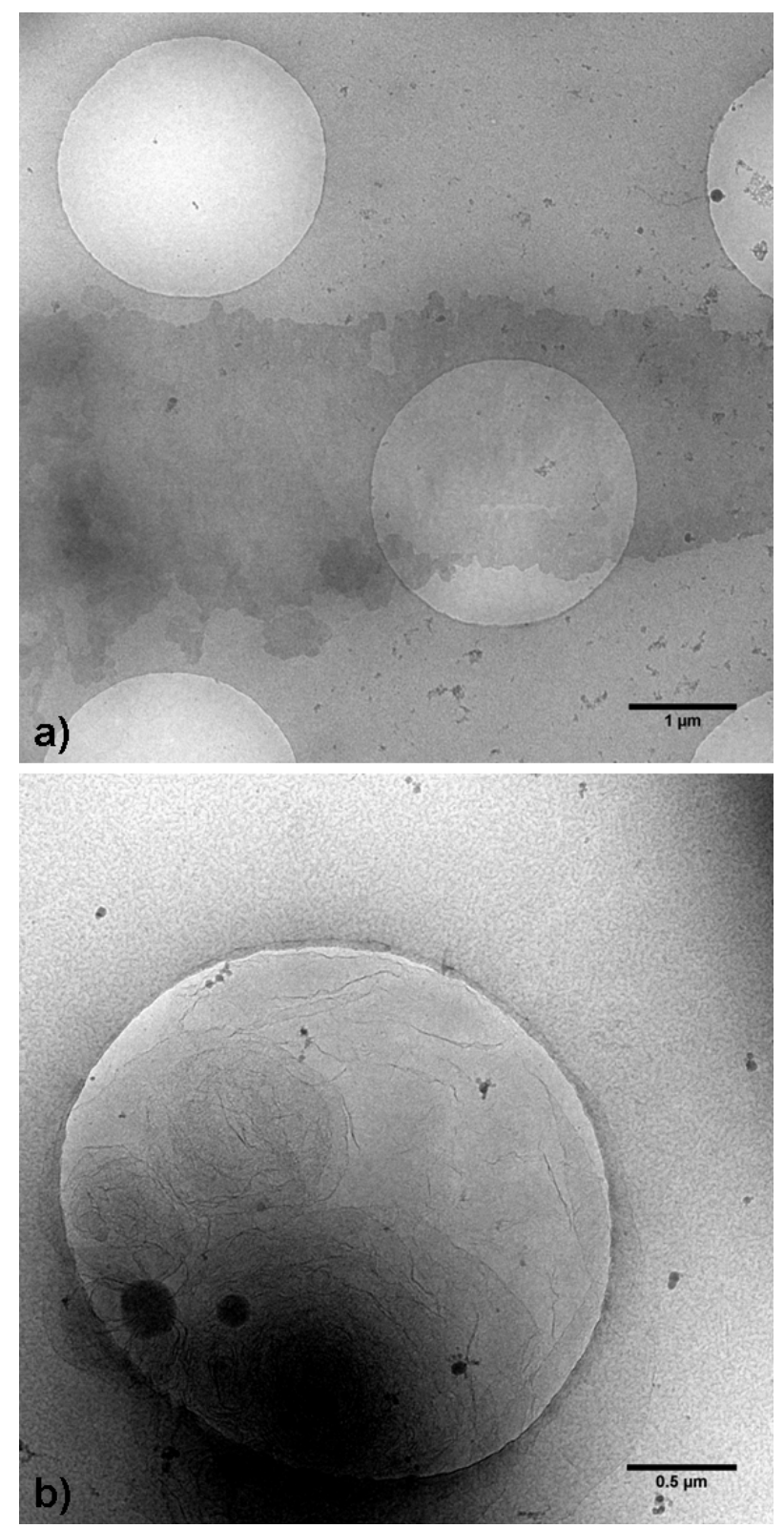

Figure 11 - Cryo-TEM images of 0.5 w\% G-C18:1 solutions prepared using the "pH-jump" technique (pH 11 --> pH 5.5) at $\mathrm{T}=5^{\circ} \mathrm{C}$, below the $\mathrm{T}_{\mathrm{M}}$ of oleic acid $\left(13^{\circ} \mathrm{C}\right)$ and stored during $24 \mathrm{~h}$. 

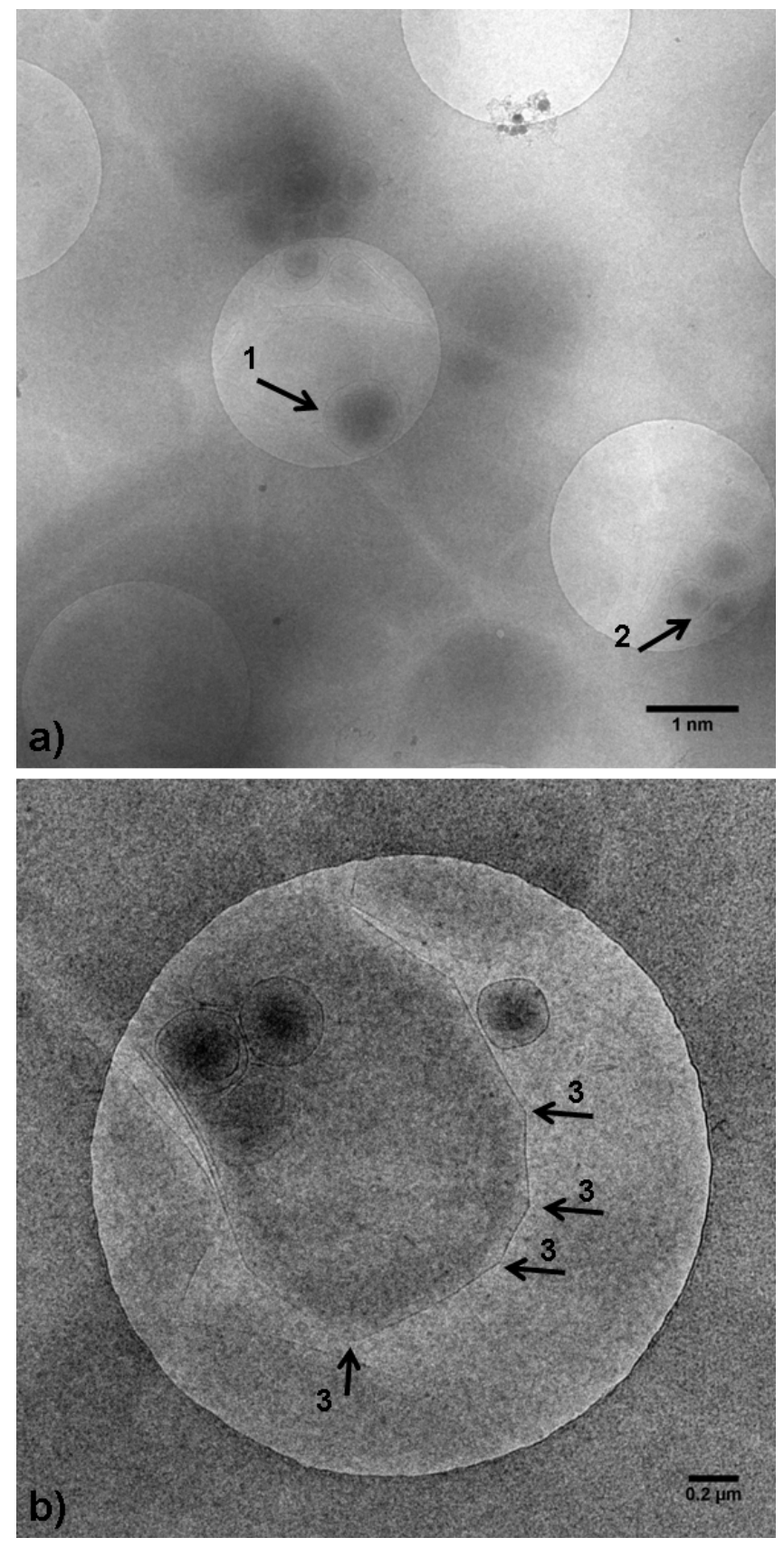

Figure 12 - Cryo-TEM images of 0.5 w\% G-C18:0 solutions prepared using the "pH-jump" technique (pH $\sim 11$--> pH 5.5) at $\mathrm{T}=80^{\circ} \mathrm{C}$ above the $\mathrm{T}_{\mathrm{M}}$ of stearic acid $\left(69^{\circ} \mathrm{C}\right)$ and stored during $24 \mathrm{~h}$. 


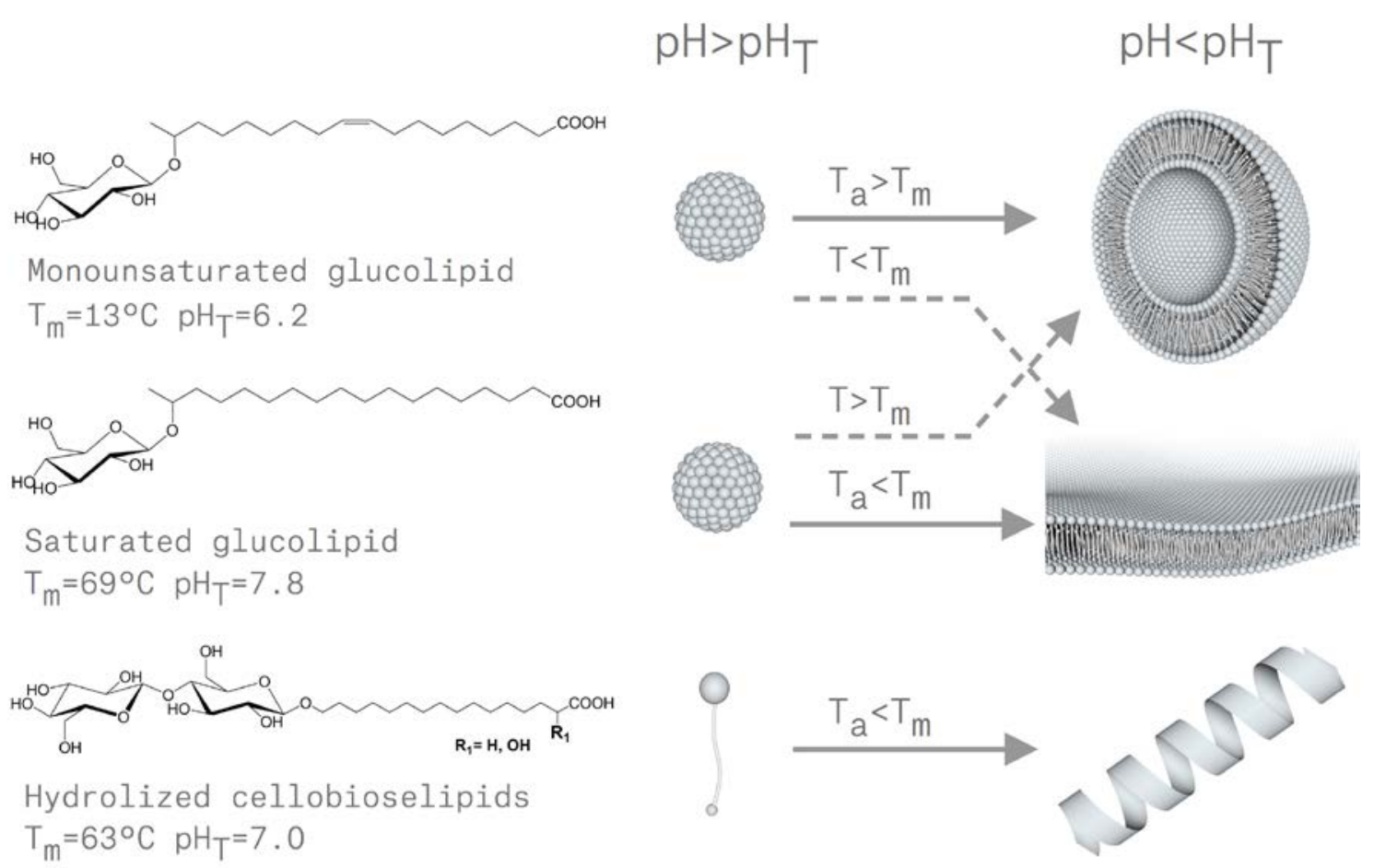

Figure 13- Scheme of the glycolipid self-assembly behaviour as a function of $\mathrm{pH}$ and temperature. $\mathrm{pH}_{\mathrm{T}}$ refers to the transition $\mathrm{pH}$ measured in Figure $2 . T, T_{a}$ and $T_{m}$ respectively refer to: the temperature, ambient (or room) temperature $\left(T_{a}=23 \pm 2^{\circ} \mathrm{C}\right)$ and the melting temperature of the fatty acid moiety alone. For simplicity, the basic pH is considered to be mainly composed of micelles (G-C18:1 and G-C18:0) or monomers (CLH). For a full description of the basic $\mathrm{pH}$ region, please ref to Figure 3 and related discussion.

\section{Conclusion}

This work deals with the pH-driven self-assembly of three microbial glycolipids in water at room temperature. G-C18:1 and G-C18:0 are composed of a single, non-acetylated, glucose unit and, respectively, an oleic and stearic acid fatty acid chain. The third one is a hydrolyzed, non-acetylated, derivative of cellobioselipids, CLH. We show that all compounds are watersoluble only at basic $\mathrm{pH}$ and become insoluble upon lowering the $\mathrm{pH}$, the exact value of which is compound-dependent. We find a transition $\mathrm{pH}$ of 6.2, 7.8 and 7.0 for, respectively, G-C18:1, G-C18:0 and CLH. Above the transition pH, G-C18:1 and G-C18:0 form a mixture of micelles and a heterogeneous mixture of, respectively, high (filamentous) and lowcurvatures (vesicles, open sheet, giant tubes) aggregates according to cryo-TEM and in-situ SAXS experiments. Interestingly, in CLH there is no trace of free micelles according to SAXS but a complex mixture of ill-defined filamentous matter. Upon decrease of $\mathrm{pH}$ below 
the transition region, G-C18:1 forms vesicles of tens and hundred of nanometers in size, GC18:0 forms infinite flat sheets and CLH mainly forms infinitely long fibers with various chiral characteristics (flat, twisted, helical). In the perspective of predicting the formation of self-assembled aggregates from functional glycolipids, we perform a semi-quantitative analysis of these results in which we show that none of these structures could have been predicted on the sole basis of the well-known packing parameter, $p$. Except the SL-C18:1 and G-C18:1 samples, for which $p$ can predict the experimental results, $p$ can be estimated to be below 0.6 for each compound, a value which is not compatible with the low-curvature structures obtained at low $\mathrm{pH}$, and for which one would expect a unitary molecular packing parameter.

To explain our results we employ additional arguments involving the temperaturedependent membrane elasticity, a concept often used to explain the type of aggregates observed in phospholipid systems. We assume that the temperature at which the experiments are performed, RT, is respectively above and below the typical melting temperature, $\mathrm{T}_{\mathrm{M}}$, of oleic $\left(13^{\circ} \mathrm{C}\right)$ and stearic $\left(69^{\circ} \mathrm{C}\right)$ acids, composing the lipophilic part of G-C18:1 and G-C18:0. At $\mathrm{T}>\mathrm{T}_{\mathrm{M}}$, one can expect flexible lipid membranes, as it could be the case of vesicles in the G-C18:1 system, having a positive mean and gaussian curvature. At $T>T_{M}$, one can reasonably expect the formation of rigid membranes, as for G-C18:0, with zero mean and gaussian curvatures. We have verified this hypothesis by performing two complementary experiments at $\mathrm{T}=5^{\circ} \mathrm{C}$ for $\mathrm{G}-\mathrm{C} 18: 1$ and $\mathrm{T}=80^{\circ} \mathrm{C}$ for G-C18:0, respectively below and above their corresponding fatty acids $T_{M}$ 's. Under these conditions, we find the expected crossstructural switch, with G-C18:1 forming now large flat sheets and G-C18:0 forming vesicles. In the end, this work not only describes the self-assembly in water of uncommon microbial glycolipids but it also provides the tools to understand the type of aggregates formed and help predict the structures of similar compounds in the future.

\section{Acknowledgements}

SAXS experiments were performed on beamline ID02 at the European Synchrotron Radiation Facility (ESRF), Grenoble, France and on the beamline SWING at the SOLEIL synchrotron Facility, Saint-Aubin, France. The research leading to these results has received funding from the European Community’s Seventh Framework Programme (FP7/2007-2013) under Grant Agreement $\mathrm{n}^{\circ}$ Biosurfing/289219. David Benqué (Protoplot, www.protoplot.com) is acknowledged for the graphic design images in Figure 13. 


\section{Supporting Information Available}

Figure S 1 shows the HPLC-ELSD chromatograms of the as-synthesized and purified monounsaturated glucolipid mixture. Figure $S 2$ contains the ${ }^{1} \mathrm{H}$ NMR spectra of G-C18:1, GC18:0 and CLH. Figure S 3 presents the LC-MS data for the as-synthesized cellobioselipid. Figure S 4 shows the ${ }^{1} \mathrm{H}_{-}{ }^{1} \mathrm{H}$ 2D COSY 45 correlation experiments recorded on the CLH sample. Figure S 5 shows the in-situ SAXS data recorded above pH 9 on G-C18:1, G-C18:0 and CLH. Figure S 6 shows the pH-dependent circular dichroism spectroscopy data for GC18:1, G-C18:0 and CLH. Figure S 7 shows the modelled SAXS curve recorded on the G$\mathrm{C} 18$ :0 sample at $\mathrm{pH}$ 6.32. Figure S 8 shows the in-situ and ex-situ SAXS data of the CLH system at acidic $\mathrm{pH}$. Figure $\mathrm{S} 9$ and Figure $\mathrm{S} 10$ respectively show complementary cryo-TEM images recorded for $\mathrm{G}-\mathrm{C} 18: 1$ at $\mathrm{T}=5^{\circ} \mathrm{C}$ and $\mathrm{G}-\mathrm{C} 18: 0$ at $\mathrm{T}=80^{\circ} \mathrm{C}$. Table $\mathrm{S} 1$ presents the composition of the G-C18:0. This information is available free of charge via the Internet at http://pubs.acs.org

\section{References}

${ }^{1}$ M. Corti, L. Cantù, P. Brocca, E. Del Favero, Self-assembly in glycolipids, Curr. Op. Coll. Interf. Sci., 2007, 12, 148-154

${ }^{2}$ G. John, M. Masuda, Y. Okada, K. Yase, T. Shimizu, Nanotube formation from Renewable Resources via Coiled Nanotubes Adv. Mater., 2001, 13, 715-718

${ }^{3}$ V. M. Garamus, G. Milkereit, S. Gerber, V. Vill, Micellar structure of a sugar based bolaamphiphile in pure solution and destabilizing effects in mixtures of glycolipids, Chem. Phys. Lett., 2004, 392, 105-109

${ }^{4}$ J. D. Desai, I. M. Banat, Microbiol. Microbial production of surfactants and their commercial potential, Molec. Biol. Rev., 1997, 61, 47-64

${ }^{5}$ T. Shimizu, Molecular Self-Assembly into One-Dimensional Nanotube Architectures and Exploitation of Their Functions, Bull. Chem. Soc. Jpn., 2008, 81, 1554-1566

6 J. H. Jung, S. Shinkai, T. Shimizu, Spectral Characterization of Self-Assemblies of Aldopyranoside Amphiphilic Gelators: What is the Essential Structural Difference between Simple Amphiphiles and Bolaamphiphiles, Chem. Eur. J. 2002, 8, 2684 - 2690

${ }^{7}$ J. Axford, The impact of glycobiology on medicine, TRENDS Immunol., 2001, 22, 2001, 237

${ }^{8}$ M. Masuda, K. Yozac, T. Shimizu, Polymorphism of monolayer lipid membrane structures made from unsymmetrical bolaamphiphiles, Carbohy. Res., 2005, 340, 2502-2509

${ }^{9}$ D. A. Frankel, D. F. O’Brien, Supramolecular Assemblies of Diacetylenic Aldonamides, J. Am. Chem. Soc. 1994, 116, 10057-10069

10 N. Kameta, H. Minamikawa, M. Masuda, Supramolecular organic nanotubes: how to utilize the inner nanospace and the outer space, Soft Matter, 2011, 7, 4539

11 D. Kitamoto, T. Morita, T. Fukuoka, M. Konishi, T. Imura, Self-assembling properties of glycolipid biosurfactants and their potential applications, Curr. Op. Coll. Interf. Sci., 2009, 14, 315-328

${ }^{12}$ G. Milkereit, V. M. Garamus, K. Veermans, R. Willumeit, V. Vill, Structures of micelles formed by synthetic alkyl glycosides with unsaturated alkyl chains, J. Coll. Interf. Sci., 2005, 284, 704-713

${ }^{13}$ L. H., V. M. Garamus, S. S. Funari, M. Malfois, R. Willumeit, B. Niemeyer, Comparison of Small-Angle Scattering Methods for the Structural Analysis of Octyl- $\beta$-maltopyranoside Micelles, J. Phys. Chem. B, 2002, 106, 7596-7604

14 S. Kamiya, H. Minamikawa, J. H. Jung, B. Yang, M. Masuda, T. Shimizu, Molecular Structure of Glucopyranosylamide Lipid and Nanotube Morphology, Langmuir 2005, 21, 743-750 743

15 S. Sonnino, A. Prinetti, L. Mauri, V. Chigorno, G. Tettamanti, Dynamic and Structural Properties of Sphingolipids, Chem. Rev. 2006, 106, 2111-2125

${ }^{16}$ R. Genc, M. Ortiz, C. K. O’Sullivan, Curvature-Tuned Preparation of Nanoliposomes, Langmuir 2009, 25, 12604-12613

${ }^{17}$ K. Bryskhe, S. Bulut, U. Olsson, Vesicle Formation from Temperature Jumps in a Nonionic Surfactant System, J. Phys. Chem. B 2005, 109, 9265-9274 
${ }^{18}$ R. Nagarajan, E. Ruckenstein Theory of Surfactant Self -Assembly: A Predictive Molecular Thermodynamic Approach, Langmuir 1991, 7, 2934-2969

${ }^{19}$ J. Leng, S. U. Egelhaaf, and M. E. Cates, Kinetics of the Micelle-to-Vesicle Transition: Aqueous Lecithin-Bile Salt Mixtures, Biophysical J., 2003, 85, 1624-1646

${ }^{20} \mathrm{~J}$. N. Israelachvili, D. J. Mitchell, B. W. Ninham, Theory of self-assembly of hydrocarbon amphiphiles into micelles and bilayers, Chem. Soc., Faraday Trans. 2 1976, 72, 1525-1568

${ }^{21}$ J. E. Klijn, M. C.A. Stuart, M. Scarzello, A.o Wagenaar, J. B. F. N. Engberts, pH-Dependent Phase Behaviour of Carbohydrate-Based Gemini Surfactants. The Effects of Carbohydrate Stereochemistry, Head Group Hydrophilicity, and Nature of the Spacer, J. Phys. Chem. B 2007, 111, 5204-5211

${ }^{22} \mathrm{~S}$. M. Gruner, Intrinsic curvature hypothesis for biomembrane lipid composition: A role for nonbilayer lipids, Proc. Nail. Acad. Sci. USA, 1985, 82, 3665-3669

${ }^{23}$ R. Strey, Microemulsion microstructure and interfacial curvature, Colloid Polym Sci, 1994, 272, 1005-1019

${ }^{24}$ P. Dhasaiyan, A. Banerjee, N. Visaveliya, B. L. V. Prasad, Influence of the Sophorolipid Molecular Geometry on their Self-Assembled Structures, Chem. - Asian J., 2013, 8, 369-372

${ }^{25}$ A.-S. Cuvier, J. Berton, C. V. Stevens, G. C. Fadda, F. Babonneau, I. N. A. Van Bogaert, W. Soetaert, G. Péhau-Arnaudet, N. Baccile, pH-triggered formation of nanoribbons from yeast-derived glycolipid biosurfactants, Soft Matter, 2014,10, 3950-3959

${ }^{26}$ H.-J. Asmer, S. Lang, F. Wagner, V. Wray, Microbial production, structure elucidation and bioconversion of sophorose lipids, J. Am. Oil. Chem. Soc., 1988, 65, 1460-1466

${ }^{27}$ N. Baccile, F. Babonneau, J. Jestin, G. Pehau-Arnaudet, I. Van Bogaert Unusual, pH-induced, self-assembly of sophorolipid biosurfactants, ACS Nano, 2012, 6, 4763-4776

${ }^{28}$ S. Manet, A.-S. Cuvier, C. Valotteau, G. C. Fadda, J. Perez, E. Karakas, S. Abel, N. Baccile, Structure of Bolaamphiphile Sophorolipid Micelles Characterized with SAXS, SANS, and MD Simulations, J. Phys. Chem. B, 2015, 119, 13113-13133

${ }^{29}$ A.-S. Cuvier, F. Babonneau, J. Berton, C. V. Stevens, G. C. Fadda, G. Péhau-Arnaudet, P. Le Griel, S. Prévost, J. Perez, N. Baccile, Nanoscale platelet formation by monounsaturated and saturated sophorolipids under basic pH conditions, Chem. - Europ. J., 2015, 21, 19265-19277

${ }_{30}$ M. Masuda, T. Shimizu, Lipid Nanotubes and Microtubes: Experimental Evidence for Unsymmetrical Monolayer Membrane Formation from Unsymmetrical Bolaamphiphiles, Langmuir, 2004, 20, 5969-5977

${ }^{31}$ S. Spoeckner, V. Wray, M. Nimtz, S. Lang, Glycolipids of the smut fungus Ustilago maydis from cultivation on renewable resources Appl. Microbiol. Biotechnol., 1999, 51, 33

${ }^{32}$ K. M. J. Saerens, J. Zhang, I. N. A. Van Bogaert, W. Soetaert, Cloning and functional characterization of the UDP-glucosyltransferase UgtB1 involved in sophorolipid production by Candida bombicola and creation of a glucolipid-producing yeast strain. Yeast, 2011, 28, 279-292.

${ }^{33}$ S. Lang, A. Brakemeier, R. Heckmann, S. Spockner, U. Rau Production of native and modified sophorose lipids. Chimica Oggi-Chemistry Today, 2000, 18, 76-79.

${ }^{34}$ S. Hewald, K. Josephs., M. Boelker., Genetic analysis of biosurfactant production in Ustilago maydis. Appl. Environ. Microbiol, 2005, 71, 3033-3040

35 M. Günther, S. Zibek, T. Hirth, S. Rupp Synthese und Optimierung von Cellobioselipiden und Mannosylerythritollipiden, 2010, Chem. Ing. Tech., 82, 1215-1221

${ }^{36}$ A.-S. Cuvier, F. Babonneau, J. Berton, C. V. Stevens, G. C. Fadda, I. Genois, P. Le Griel, G. Péhau-Arnaudet, N. Baccile, Synthesis of Uniform, Monodisperse, Sophorolipid Twisted Ribbons, Chem. Asian J. 2015, 2015, 10, 2419-2426

${ }^{37}$ Two-Dimensional NMR Spectroscopy - Applications for Chemists and Biochemists, Second Edition, Eds W. R. Croasmun, R. M. K. Carlson, 1994, VCH Publishers Inc.

${ }^{38}$ R. Netrabukkana, K. Lourvanij, G. L. Rorrer, Diffusion of Glucose and Glucitol in Microporous and Mesoporous Silicate/Aluminosilicate Catalysts, Ind. Eng. Chem. Res. 1996, 35, 458-464

${ }^{39}$ L. Ziserman, H.-Y. Lee, S. R. Raghavan, A. Mor, and D. Danino, Unraveling the mechanism of nanotube formation by chiral self-assembly of amphiphiles J. Am. Chem. Soc., 2011, 133, 2511-2517

${ }^{40}$ T. G. Barclay, K. Costantopoulos, J. Matisons, Nanotubes Self-Assembled from Amphiphilic Molecules via Helical Intermediates, Chem. Rev., 2014, 114, 10217-10291

${ }^{41}$ M. L. Chen, J. Penfold, R. K. Thomas, T. J. Smyth, A. Perfumo, R. Marchant, I. M. Banat, P. Stevenson, A. Parry, I. Tucker, I. Grillo, Solution self-assembly and adsorption at the air-water interface of the monorhamnose and dirhamnose rhamnolipids and their mixtures, Langmuir, 2010, 26, 18281-18292.

${ }^{42}$ R. Lieckfeldt, J. Villalain, J.-C. Gomez-Fernandez, G. Lee, Apparent pKa of the fatty acids within ordered mixtures of model human stratum corneum lipids, Pharma. Res., 1995, 12, 1614-1617

${ }^{43}$ J. R. Kanicky, D. O. Shah, Effect of Degree, Type, and Position of Unsaturation on the pKa of Long-Chain Fatty Acids, J. Colloid Interf. Sci., 2002, 256, 201-207

${ }^{44}$ S. Salentinig, L. Sagalowicz, O. Glatter, Self-Assembled Structures and pKa Value of Oleic Acid in Systems 
of Biological Relevance, Langmuir 2010, 26, 11670-11679

45 J. R. Kanicky, A. F. Poniatowski, N. R. Mehta, D. O. Shah Cooperativity among Molecules at Interfaces in Relation to Various Technological Processes: Effect of Chain Length on the pKa of Fatty Acid Salt Solutions, Langmuir 2000, 16, 172-177

${ }^{46}$ C. Cecutti, B. Focher, B. Perly, T. Zemb, Glycolipid Self-Assembly: Micellar Structure. Langmuir 1991, 7, $2580-2585$

${ }^{47}$ Geometric Methods in the Elastic Theory of Membranes in Liquid Crystal Phases, Chapter 1, By O.-Y. Zhong-Can, L. Ji-Xing, X. Yu-Zhang Eds., World Scientific Publishing, Singapore, 1999

${ }^{48}$ L. Ziserman, A. Mor, D. Harries, D. Danino, Curvature instability in a chiral amphiphile self-assembly, Phys. Rev. Lett., 2011, 106, 238105

${ }^{49}$ A. Walter, P. K. Vinson, A. Kaplun, Y. Talmon, Intermediate structures in the cholate-phosphatidylcholine vesicle-micelle transition, Biophys. J., 1991, 60, 1315-1325

50 M. C.A. Stuart, E. J. Boekema, Two distinct mechanisms of vesicle-to-micelle and micelle-to-vesicle transition are mediated by the packing parameter of phospholipid-detergent systems, Biochim. Biophys. Acta, 2007, 1768, 2681-2689 


\section{Supporting Information}

\section{List of Figure Caption}

Figure S 1 - HPLC-ELSD chromatograms of (A) glucolipid fermentation product with hydrophilic and hydrophobic contaminants derived from a $1.5 \mathrm{~L}$ experiment and used for alkaline hydrolysis and (B) nonacetylated glucolipids after alkaline hydrolysis and tBME extraction used in this work (mainly G-C18:1).

Figure S 2 - ${ }^{1} \mathrm{H}$ NMR spectra of a) G-C18:1 and G-C18:0; b) CLH

Figure S 3 - LC-MS data for the as-synthesized cellobioselipid (CL) compound

Figure S $4-{ }^{1} \mathrm{H}-{ }^{1} \mathrm{H}$ 2D COSY 45 correlation experiments recorded on the CLH sample. a) and d) simply highlight different correlation pathways in a single COSY45 experiment in CLH samples with respectively $R_{1}=H$ and $R_{1}=O H$. Extract of a long-range TOCSY experiment is presented as inset in (b).

Figure S 5 - in-situ SAXS data recorded in the $11.5<$ pH $<9$ range on glucolipids G-C18:1, G-C18:0 and hydrolyzed cellobioselipids, CLH

Figure S 6 - Circular Dichroism spectroscopy recorded on 5 mg/mL solutions of CLH, G-C18 :0 and G$\mathrm{C} 18$ :1 samples as a function of $\mathrm{pH}$

Figure S 7 - SAXS curve recorded on the G-C18 :0 sample at pH 6.32 and fitted with a core-shell bicelle form factor model function using the SasView 3.0.0 software version

Figure S 8 - a) pH-resolved in-situ (ID02 line, ESRF synchrotron, Grenoble, France) and b) ex-situ SAXS experiments recorded on the CLH sample. Pattern at $\mathrm{pH}<4$ has been recorded on the SWING line, SOLEIL synchrotron, Saint-Aubin, France. Pattern at pH $\sim 6$ has been recorded on a laboratory SAXS instrument with a sample-to-detector distance of $843 \mathbf{~ m m}$

Figure S 9 - Additional cryo-TEM images of 0.5 w\% G-C18:1 solutions prepared using the “pH-jump” technique (pH 11 --> pH 5.5) at $\mathrm{T}=5^{\circ} \mathrm{C}$, below the $\mathrm{T}_{M}$ of oleic acid $\left(13^{\circ} \mathrm{C}\right)$ and stored during $24 \mathrm{~h}$.

Figure S 10 - Additional cryo-TEM images of 0.5 w\% G-C18:0 solutions prepared using the “pH-jump” technique (pH 11 --> pH 5.5) at $\mathrm{T}=80^{\circ} \mathrm{C}$ above the $\mathrm{T}_{M}$ of stearic acid $\left(69^{\circ} \mathrm{C}\right)$ and stored during $24 \mathrm{~h}$.

\section{List of Table Caption}

Table S 1: Main non-acetylated acidic glucolipid and relative abundance (based on peak area derived 
from chromatogram Figure S 1B). In C18: $X, X$ identifies the number of unsaturation in the fatty acid. Terminal and subterminal refer to the position of glucose with respect to the C17 (subterminal) or C18 (terminal) position of the fatty acid chain ( $\mathrm{C} 1$ being the $\mathrm{COOH}$ group). 
Table of Content

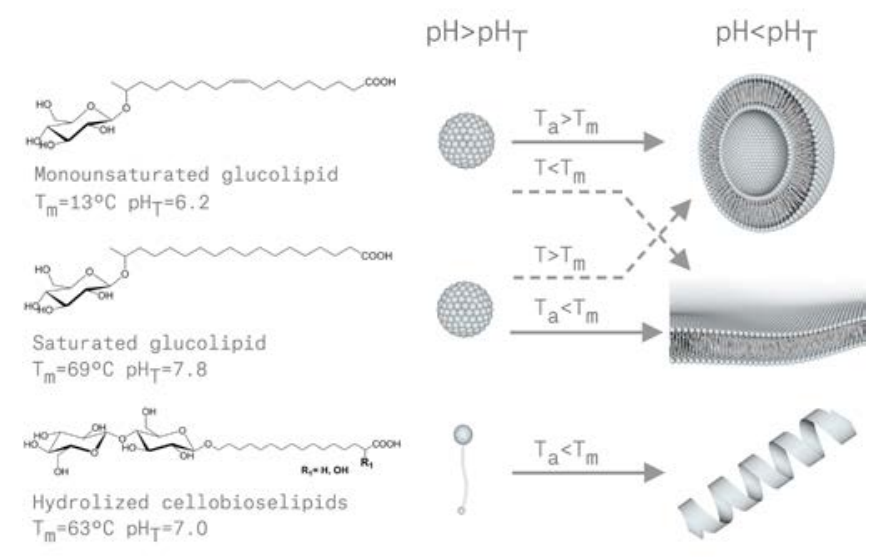

